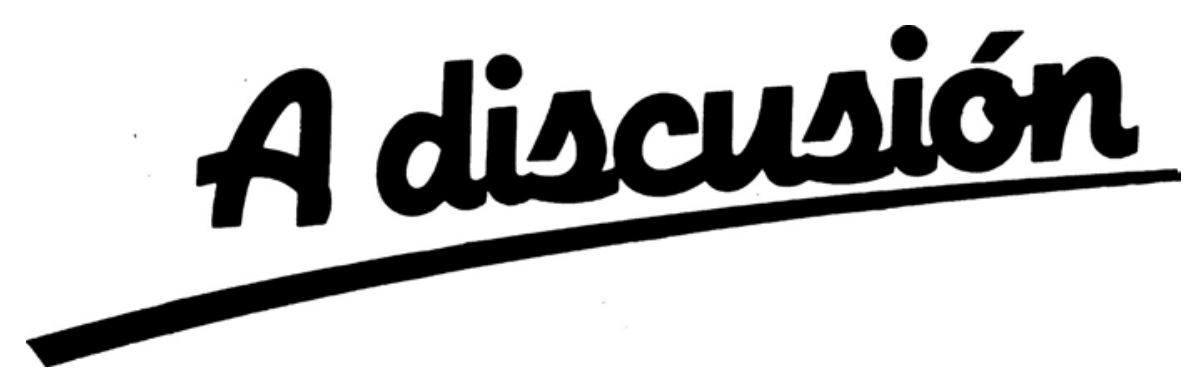

\title{
NASH EQUILIBRIUM IN A MODEL OF MULTIPRODUCT PRICE COMPETITION: AN ASSIGNMENT PROBLEM*
}

\section{Ivan Arribas and Amparo Urbano**}

WP-AD 2003-21

Correspondence: Universitat de València. Departamento de Análisis Económico. Campus dels Tarongers. Edificio Departamental Oriental. Avda. dels Tarongers, s/n. 46022 Valencia. Tel. 34963828207 / fax 34-96 38282 49. e-mail: Amparo.Urbano@uv.es and Ivan.Arribas@uv.es.

Editor: Instituto Valenciano de Investigaciones Económicas, S.A.

Primera Edición Junio 2003

Depósito Legal: V-2867-2003

IVIE working papers offer in advance the results of economic research under way in order to encourage a discussion process before sending them to scientific journals for their final publication.

\footnotetext{
* The authors thank financial support from the Spanish Ministry of Science and Technology under project B2000-1429.

** Universitat de València.
} 


\title{
NASH EQUILIBRIA IN A MODEL OF MULTIPRODUCT \\ PRICE COMPETITION: AN ASSIGNMENT PROBLEM
}

\author{
Ivan Arribas and Amparo Urbano
}

\begin{abstract}
We study the market interaction of a finite number of single-product firms and a representative buyer, where the buyer consumes bundles of these goods. The buyers' value function determines their willingness to pay for subsets of goods. We show that subgame perfect Nash-equilibrium outcomes are solutions of the linear relaxation of an integer programming assignment problem and that they always exits. The (subgame perfect) Nash-equilibrium price set is characterized by the Pareto frontier of the associated dual problem's projection on the firms' price vectors. We identify the Nash-equilibrium prices for monotonic buyers' value functions and, more importantly, we show that some central solution concepts in cooperative game theory are (subgame perfect) equilibrium prices of our strategic game.
\end{abstract}

Keywords: Multiproduct price competition, Integer Programming, Subgame Perfect Nash Equilibria.

JEL Classification Numbers: C72, D21, D41, D43, 13. 


\section{Introduction.}

We study the market interaction of a finite number of firms and a representative buyer, where each firm produces at most one good and the buyer consumes bundles of these goods. We show that subgame perfect Nashequilibrium outcomes are solutions of the linear relaxation of an integer programming assignment problem, where subsets of goods are assigned to the buyer.

Strategic interaction in markets has been thoroughly studied in the literature, and has mostly focused on the case of several firms which produce a homogeneous good or goods which are close substitutes. The most well known works are the Cournot quantity and the Bertrand price competition models. However, the scenario where firms produce goods of a very general nature has received little attention.

The literature on strategic multiproduct oligopolistic competition deals with product differentiation, where each firm produces a single good with different characteristics, address models. Here we find models of spatial competition, where firms differ in their location (see Hotelling 1929; d'Aspremont et al. 1979; Salop 1979, among others) and models of quality differentiation where a number of firms produce substitute goods that differ in quality and then sell to a continuum of buyers, identical in tastes but different in income (e.g. Gabszewicz and Thisse 1979, 1980, and Shaked and Sutton 1982, 1983). In address models, firms try to relax competition through product differentiation either by increasing distance from rival firms or by considering quality gaps. On the other hand, the existence of pure strategy equilibrium in a standard multiproduct Bertrand competition, has been shown by Milgrom and Roberts (1990) and Milgrom and Shanon (1994), under some restrictions on the demand and cost functions, and when all goods are only either substitutes or complements.

A different approach is that of Tauman et al. (1997, TUW, hereafter) who study a simple model of price competition in a multiproduct oligopoly market where goods can be of a very general nature and not necessarily substitutes or complements. Each firm produces only one good and buyers are all identical. The (representative) buyer purchases a bundle of goods and either one or zero units of each of the products are chosen. Moreover, she is characterized by her willingness to pay for every subset of goods. Firms are engaged in price competition in the first stage and buyers take their 
consumption decisions in the second stage. They show that a pure strategy equilibrium always exists and that the equilibrium consumption is always efficient in the sense of maximizing total social surplus. The equilibrium prices depend on the buyer's value function, i.e. the buyer's willingness to pay as a function of the consumption bundle. However, the price function can only be characterized either under concavity or convexity of the buyer's value function.

Our work generalizes the analysis of TUW(1997) and characterizes the set of all subgame perfect Nash-equilibrium outcomes. Since the demand function is unitary and buyers purchase bundles of goods, the equilibrium analysis leads us towards combinatorial complexity which makes it necessary to use integer linear programming to better handle the model's indivisibilities. Specifically, we offer the set of equilibrium prices as some solutions of the associated dual to the linear relaxation of an integer programming assignment problem.

We show that the solutions of this linear programming problem are the subgame perfect Nash-equilibrium prices and consumption set. It is interesting to note that the solutions of a linear programming problem are a convex polyhedron, and so is the projection of the dual problem's solutions on firms' price vectors. This polyhedron is completely determined by its vertices. The Pareto frontier of the above projection has to be identified in order to characterize the set of all (subgame perfect) Nash-equilibrium price vectors. As this frontier can be expressed as the convex combination of non-Pareto dominated vertices, we just need to obtain all these vertices. At every Nash equilibrium, a non-active firm may set prices equal to or different from marginal costs, and the selected firms' prices are the non-Pareto dominated vectors of the above Pareto frontier. The equilibrium consumption set is always efficient. Moreover, equilibrium prices belong to the core of the economy and, since in this model the core is always decentralized by Walrasian prices, the Nashequilibrium prices are a subset of them. Thus, a first important contribution is to offer a non-cooperative framework which provides foundations for cooperative solutions as the result of strategic behavior.

Furthermore, when the buyers' value function is convex, symmetric $k$ convex or 1-convex, some specific solution concepts in cooperative game theory, such as the Shapley value and the nucleolus of the buyer's value function are subgame perfect Nash-equilibrium prices of our non-cooperative game. In general, when the buyer's value function is $k$-convex, her surplus is always 
zero and then the core of this value function is always priced as subgame perfect Nash-equilibrium prices. These results are related with the literature on the design of simple non-cooperative mechanisms implementing cooperative solutions concepts as in Pérez-Castrillo (1994), Pérez-Castrillo and Wettstein (2001) and Pérez-Castillo and Sotomayor (2002), among others.

When the buyer's value function for bundles of goods is monotonic and products are substitutes, then equilibrium prices are products' marginal contributions and the buyer's surplus is always positive, which reflects the underlying market competition. Moreover, under this setting the equilibrium price vector is unique and coincides with the maximal Walrasian price of the economy. In other words, the most preferred point by firms (or the least preferred by the buyer) in the core of the economy is priced as the subgame perfect Nash-equilibrium price vector. Now it is easy to show that when the buyer's value function is concave, then products are substitutes and the above result applies.

The market model considered here is also related to the matching literature (e.g. Kelso and Crawford 1982, KC) and with assignment games. In particular, some extensions of the canonical standard assignment model, with many sellers and buyers interacting, have recently received increasing attention. These models are two-sided matching markets, where sellers have an initial endowment of indivisible objects and buyers have a utility function over any package or bundle of objects. Differences in the framework are based on the units produced (each seller has only one product and only one unit of this product or sellers have no restriction on their production); the units purchased (just one or a bundle); the number of sellers (one or several); the number of buyers; the price of a bundle (additive or non-additive pricing functions), etc. The package assignment problem has been studied by Gul and Stacchetti (2000, GS), Bikhchandani and Mamer (1997, BM) and Bikhchandani and Ostroy (2001, BO), among others. In all these papers utility functions are quasilinear in money, defined on bundles of goods and buyers play the same role: they select, given firms' prices, the best bundle. The main difference between these models and our paper is that we deal with strategic equilibrium where firms are price setters, while they deal with Walrasian equilibria. Another difference is that we deal with homogeneous buyers, but no restriction on the set of goods is assumed, while, for instance, GS deal with heterogeneous buyers, but with goods which have no complementarities (a notion closely related to gross substitutability). BM and BO 
give a linear programming characterization of the Walrasian equilibrium outcomes while we provide it for subgame perfect Nash-equilibrium outcomes, although their model is more general than ours.

To sum up, we formulate the subgame perfect Nash-equilibrium outcomes of a multiproduct market with a representative buyer, as a modified extension of the standard assignment model and prove the equivalence of linear programming solutions and subgame perfect Nash-equilibrium outcomes. Although the results of this paper are driven by the fact that there is only one type of buyer, they open the door for the application of duality methods in more general strategic models.

The paper is organized as follows. The model is presented in Section 2. The assingment problem and its linear relaxation are offered in Section 3. Section 4 is devoted to prove the existence and characterization of subgame perfect Nash-equilibrium outcomes. Specific results for monotonic, concave and $k$-convex value functions are given in Section 5. Finally, Section 6 deals with the extension to positive marginal costs.

\section{The model}

The model consists of a buyer (or a set of identical buyers) and $n$ firms as in TUW (1997). Each firm produces one unit of a single product. Moreover, different firms may produce different goods. Let $N=\{1,2, \ldots, n\}$ be the set of firms. We use the same notation for firms and products. The buyer consumes either one or zero units of each one of the $n$ products and is denoted as player 0 .

A consumption set of the buyer is a subset $S$ of $N$. The buyer has a value function $v(S)$ over any subset $S \subseteq N$, which represents her total willingness to pay for the consumption set $S$, with ${ }^{1} v(\emptyset)=0$. Let $c_{i}$ be the (constant) unit cost of production of firm $i$ and $c=\left(c_{1}, c_{2}, \ldots, c_{n}\right)$ the cost vector.

The sequence of events unfolds as follows. First, each firm $i$ chooses its price $p_{i} \in R_{+}$independently and simultaneously to the others. Then, the buyer observes the price vector $p=\left(p_{1}, \ldots, p_{n}\right) \in R_{+}^{n}$ and selects a consumption set $S \subseteq N$ as a function of $p$. As a result of the trade each firm $i$ in $S$

\footnotetext{
${ }^{1}$ The function $v$ can be derived from a consumer's utility function which is quasilinear in money. Then, $v: 2^{N} \longrightarrow R$, where $2^{N}$ is the power set of $N$.
} 
earns a net payoff of $p_{i}-c_{i}$ and zero otherwise. The buyer obtains a payoff equal to her surplus.

Formally, we have a strategic game with $n+1$ players, $0,1,2, . ., n$. The set of strategies of each firm is $R_{+}$and that of the buyer is $S_{0}$, the set of functions $\underline{S}$ from $R_{+}^{n}$ to $2^{N}$. Finally, the payoff function (the profit) for each $i \in N$ is given by

$$
\pi_{i}(\underline{S}, p)=\left\{\begin{array}{cc}
p_{i}-c_{i} & i \in \underline{S}(p) \\
0 & i \notin \underline{S}(p)
\end{array}\right.
$$

where $\underline{S}(p)$ is the consumption set of the buyer corresponding to $p \in R_{+}^{n}$. The payoff function of the buyer is given by her surplus $c s(\underline{S}, p)=v(\underline{S}(p))-$ $\sum_{k \in \underline{S}(p)} p_{k}$.

Denote this economy by $G(n+1, v, c)$ and let $S P E^{*}$ be the set of all its pure strategy subgame perfect equilibria. Let $(S, p)$ be in $S P E^{*}$. Then $p$ is called an $S P E^{*}$-price vector, $S=S(p)$ an $S P E^{*}$-consumption set and $(S, p)$ an $S P E^{*}$-outcome.

It is not difficult to show that $(N, p)$ is an $S P E^{*}$-outcome iff $p \geq c$ and

(CB) Buyer optimality: $v(N)-\sum_{k \in N} p_{k} \geq v(T)-\sum_{k \in T} p_{k}$ for all $T \subseteq N$;

(CF1) Firm optimality: for every $i \in N$, there is $S_{i} \subseteq N \backslash\{i\}$ such that,

$$
v(N)-\sum_{k \in N} p_{k}=v\left(S_{i}\right)-\sum_{k \in S_{i}} p_{k}
$$

This is so because (CB) is implied by subgame perfection and (CF1) by firms' incentives. Suppose that (CF1) does not hold, then by (CB) for some $i \in N$, and for every $S_{i} \subseteq N \backslash\{i\}$,

$$
v(N)-\sum_{k \in N} p_{k}>v\left(S_{i}\right)-\sum_{k \in S_{i}} p_{k},
$$

and then firm $i$ is better off charging a price $p_{i}+\varepsilon$, for a sufficiently small $\varepsilon>0$, such that (CB) is still satisfied for all $S_{i} \subseteq N \backslash\{i\}$. This implies that the buyer, when observing the price vector $\left(p_{-i}, p_{i}+\varepsilon\right)$, will again choose the consumption set $N$. Conversely, if (CB) and (CF1) are satisfied then $(N, p)$ is an $S P E^{*}$-outcome since $N$ is a best choice for the buyer and no firm has an incentive to either reduce or increase its price. Notice that set $S_{i}$ in $(\mathrm{CF} 1)$ may be empty and in this case $v(N)-\sum_{k \in N} p_{k}=0$, and firms extract the entire buyer surplus. 
Suppose now that $(S, p)$ is an $S P E^{*}$-outcome with $S \neq N$. Then, the equilibrium conditions must guarantee that no firm $i$ outside $S$ benefits from a price reduction and thus $S$ has to remain a best choice for the buyer even if a firm $i \notin S$ reduces its price to its marginal cost level. Then, $(S, p)$ is an $S P E^{*}$-outcome iff $p_{k} \geq c_{k}$ for every $k \in S,(\mathrm{CB})$ and $(\mathrm{CF} 1)$ are satisfied in $S$, and

(CF2) for every $i \notin S$, and for every $T \ni i$,

$$
v(S)-\sum_{k \in S} p_{k} \geq v(T)-\sum_{k \in T \backslash i} p_{k}-c_{i} .
$$

As in TUW(1997), we only consider the set of pure strategy subgame perfect equilibria of the above economy which remains as equilibrium outcomes even if all non-selling firms set marginal cost prices. This restriction removes the set of equilibrium outcomes in which firms charge unreasonably high prices so that no individual firm can benefit from a price reduction of its product (see example 1 below). To define this restriction of the set of Nash equilibria consider $p=\left(p_{1}, \ldots, p_{n}\right) \in R_{+}^{n}$ and let $S \subseteq N$. Denote by $p^{S}$ the vector in $R_{+}^{n}$ such that

$$
p^{S}=\left\{\begin{array}{cc}
p_{i} & i \in S \\
c_{i} & i \notin S
\end{array}\right.
$$

Definition 1 For every triplet $(N, v, c)$ define

$$
S P E=\left\{(S, p) \in S P E^{*} \mid p \geq c \text { and }\left(S, p^{S}\right) \in S P E^{*}\right\}
$$

Assume for simplicity that each $c_{i}=0, i \in N$ (we will consider the nonzero cost case in the last section). The next Proposition characterizes the set of $S P E$-outcomes (for $c=(0,0, \ldots, 0)$ ), where $(\mathrm{C} 1)$ and $(\mathrm{C} 2)$ are as $(\mathrm{CB})$ and $(\mathrm{CF} 1)$ respectively, and $(\mathrm{C} 3)$ refers now to sets of non-selected firms instead of (CF2) (its proof runs similar to that of (CB), (CF1) and (CF2)).

Throughout the paper we denote by $|S|$ the number of products (firms) in the consumption set $S \subseteq N$.

Proposition 1 (TUW,1997) (1) Suppose that $S \neq N$. Then $(S, p)$ is an $S P E$-outcome iff $p \geq 0$ and

(C1) $v(S)-\sum_{k \in S} p_{k} \geq v(T)-\sum_{k \in T} p_{k}$ for all $T \subseteq N$; 
(C2) for every $i \in S$, there is $S_{i} \subseteq N \backslash\{i\}$ such that,

$$
v(S)-\sum_{k \in S} p_{k}=v\left(S_{i}\right)-\sum_{k \in S_{i}} p_{k}
$$

(C3) for every $A \subseteq N \backslash S$ and for all $T \supseteq A$

$$
v(S)-\sum_{k \in S} p_{k} \geq v(T)-\sum_{k \in T \backslash A} p_{k} .
$$

(2) If $S=N$ then $(S, p)$ is an SPE-outcome iff $p \geq 0$ and C1-C2 are verified.

Finally, given $K \subseteq N$, define,

$$
V(K)=\max _{S \subseteq K}\{v(S)\}
$$

as the maximum social surplus in economy $G(k+1, v)=G(k+1, v, c=$ $(0,0, \ldots, 0))$, where $k=|K|$, i.e., the maximum of the buyer's value function when considering consumption sets in $K$.

Since we are interested in the "efficiency" of $S P E$-outcomes, we will compare them with the core:

Definition 2 (T-core) Let $T \subseteq N$. The $T$-core of the economy $G(n+1, v)$, denoted by $T$-core $(G)$, are all the pairs $\left(p^{b}, p\right) \in R_{+}^{n+1}$, such that

(i) $p^{b}+\sum_{k \in T} p_{k}=v(T)$,

(ii) $p^{b}+\sum_{k \in S \cap T} p_{k} \geq v(S), \quad \forall S \subseteq N$.

The element $p^{b}$ is the buyer surplus and each $p_{k}$ the payoff to firm $k$. If $T=N$, then we obtain the core of the economy, denoted core $(G)$. Also, the subset of points in the $T$-core $(G)$ such that the buyer surplus, $p^{b}$, is zero, defines the $T$-core of $v$ or $T$-core $(v)$,

$$
T \text {-core }(v)=\left\{p \in R_{+}^{n} \mid \sum_{k \in T} p_{k}=v(T), \quad \sum_{k \in S \cap T} p_{k} \geq v(S), \quad \forall S \subseteq N\right\}
$$

The intuition of the $T$-core $(v)$ concept is as follows. Suppose that the equilibrium consumption set is $T$. Then, firms in $N \backslash T$ obtain zero and hence 
will be willing to join the set of seller firms. Hence, every subset $S \subseteq T$ can actually achieve $u_{T}(S)$ where,

$$
u_{T}(S)=\max _{A \subseteq N \backslash T}\{v(S \cup A)\}
$$

It can be verified that the projection of the $T$-core $(v)$ on $T$ coincides with the $N$-core $\left(u_{T}\right)$ when the buyer surplus is zero.

\subsection{Some motivating examples}

ExAMPLE 1: The market of two right-hand gloves and one left-hand glove. Let $N=\{1,2,3\}, c_{i}=0$, for $i=1,2,3$. Firm 3 is the left-hand glove producer, and

$$
v(S)= \begin{cases}1 & 3 \in S, \quad|S| \geq 2 \\ 0 & \text { otherwise }\end{cases}
$$

SPE-outcome set: There are three $S P E$-consumption sets, $N,\{1,3\}$ and $\{2,3\}$. The prices supporting each of them are, $p=(0,0,1)$ for the first consumption set; $p=(0, \alpha, 1)$ with $\alpha \geq 0$ for the second one and $p=(\alpha, 0,1)$ for the third set. Hence, the $S P E$-outcome set is:

$$
\{(\{1,2,3\},(0,0,1))\} \cup\{(\{1,3\},(0, \alpha, 1)) \mid \alpha \geq 0\} \cup\{(\{2,3\},(\alpha, 0,1)) \mid \alpha \geq 0\}
$$

Note that the buyer surplus is zero at each equilibrium outcome, since firm 3 exploits the buyer's entire willingness to pay. Moreover, non-selected firms may fix a positive price. Every $S P E$-consumption bundle, $S$, is efficient in the sense that $S \in \arg \max _{S \subseteq N}\{v(S)\}$.

Observe that our characterization of the Nash-equilibrium rules out outcomes of the $S P E^{*}$ set. For instance, $\{\emptyset,(\beta, \beta, \beta)\}$, with $\beta>1$, belongs to the $S N E^{*}$-outcome set, since it satisfies (CB), (CF1) and (CF2). However, this equilibrium is unreasonable since no product is sold and no individual firm can sell its product by a price reduction.

The core: The set of vectors in $N$-core $(G)=\operatorname{Core}(G)$ is

$$
\operatorname{Core}(G)=\{1-\alpha,(0,0, \alpha) \mid 0 \leq \alpha \leq 1\} .
$$

Thus, the buyer surplus is $p^{b}=1-\alpha$, which is zero iff $\alpha=1$, hence the $N$-core $(v)$ is point $(0,0,1)$. 
ExAmple 2: Let $N=\{1,2,3\}, c_{i}=0$, for each $i=1,2,3$ and assume that

$$
v(S)= \begin{cases}0 & |S| \leq 1 \\ 1.5 & S=\{1,2\} \\ 1 & \text { otherwise }\end{cases}
$$

$S P E$ set: There exists a unique subgame perfect Nash equilibrium outcome of this game, $(S, p)=(\{1,2\},(0.5,0.5,0))$. The buyer surplus is positive and equal to $v(\{1,2\})-p_{1}-p_{2}=0.5$. Firm 3 does not sell its product and then the price of product 3 is equal to zero. The $S P E$-consumption set is efficient.

The core: Given that $T=\{1,2\}$, the set of price vectors in $T$-core $(G)$ is $\{(\alpha, \beta, 0) \mid 0 \leq \alpha \leq 0.5,0 \leq \beta \leq 0.5\}$. Note that since the buyer surplus is $p^{b}=1.5-\alpha-\beta \geq 0.5$, the $T$-core $(v)=\emptyset$.

ExAmple 3: This example is a variation of the one above. Let $N=$ $\{1,2,3,4\}$, and again $c_{i}=0$ for each $i=1,2,3$, with

$$
v(S)= \begin{cases}0 & |S| \leq 1 \text { or } S=\{3,4\} \\ 1.5 & S=\{1,2\} \\ 1 & \text { otherwise }\end{cases}
$$

The $S P E$-outcome set is $\{(\{1,2\},(0.5,0.5,0, \alpha)) \mid \alpha \geq 0\} \cup\{(\{1,2\},(0.5,0.5, \alpha, 0)) \mid \alpha \geq$ $0\}$. This example shows that when there is more than one firm out of the $S P E$-consumption set, at least one of them charges a zero price.

The core: As in example 2, since $T=\{1,2\}$, the set of vectors in $T$ $\operatorname{core}(G)$ is $\{(1.5-\alpha-\beta),(\alpha, \beta, 0,0) \mid 0 \leq \alpha \leq 0.5,0 \leq \beta \leq 0.5\}$ and the $T$-core $(v)=\emptyset$.

EXAMPLE 4: In the above examples the buyer surplus is the same in all $S P E$-outcomes. However, this is not true in general as shown below. Let $N=\{1,2,3\}, c=(0,0,0)$ and let the value function be,

\begin{tabular}{c|ccccccc}
$S$ & 1 & 2 & 3 & 1,2 & 1,3 & 2,3 & $1,2,3$ \\
\hline$v$ & 6 & 5 & 5 & 0 & 0 & 0 & 10
\end{tabular}

$S P E$ set: The SPE-outcomes are,

$$
\begin{aligned}
S P E= & \{(N,(5-\alpha, 4-\alpha, \alpha)) \mid 2 \leq \alpha \leq 4\} \cup \\
& \{(N,(5-\alpha, \alpha, \alpha)) \mid 0 \leq \alpha \leq 2\} \cup \\
& \{(N,(3-\alpha, 2+\alpha, 2-\alpha)) \mid 0 \leq \alpha \leq 2\}
\end{aligned}
$$


thus, $\sum_{k \in N} p_{k}$ is not a constant. It depends on $\alpha$ and varies between 5 and 7. The buyer surplus varies from 3 , in $(N,(3,2,2))$ to 5 , in $(N,(5,0,0))$.

The Core: Notice that the $T$-core $=\operatorname{Core}(G)$ and the set of core vectors is

$\operatorname{Core}(G)=\{(10-\alpha-\beta-\gamma),(\alpha, \beta, \gamma) \mid 0 \leq \alpha+\beta \leq 5,0 \leq \alpha+\gamma \leq 5,0 \leq \beta+\gamma \leq 4\}$

and since, $p^{b}=10-\alpha-\beta-\gamma \geq 1$, then the $N$-core $(v)=\emptyset$.

\section{The Linear Programming problem}

Our main result shows that the $S P E$-outcome set of $G(n+1, v)$ is equivalent to integer-valued solutions of a corresponding linear programming "many-toone" assignment problem, LP hereafter, where subsets of goods are assigned to the buyer.

Since our purpose is to find a "suitable" assignment of a set of sellers (products) to the representative buyer, define variable $y(S)$, for each set $S \subseteq N$, which is equal to 1 if the buyer chooses the consumption set $S$ and 0 otherwise. The integer linear programming under consideration, denoted ILP is given by,

$$
\begin{aligned}
V(N)=\operatorname{Max} & \sum_{S \subseteq N} v(S) y(S) \\
\text { s.t. } & \sum_{S \subseteq N} y(S) \leq 1 \\
& \sum_{S \ni i} y(S) \leq 1 \quad \forall i \in N \\
& y(S) \in\{0,1\}
\end{aligned}
$$

The first constraint ensures that only one consumption set is selected. The next constraints are redundant given the first one, but they define the price vector in the associated dual problem. Let us consider the linear relaxation LP of ILP in which we change the integrity constraints $y(S) \in\{0,1\}$ of ILP by $y(S) \geq 0$ for all $S \subseteq N$.

Let DLP be the dual of LP. Since the right-hand sides of all the constraints are equal to one, the objective function in the DLP is, 


$$
\operatorname{Min} \pi^{b}+\sum_{i \in N} \pi_{i}
$$

where $\pi^{b}$-the variable associated to the first constraint- stand for the buyer's surplus and $\pi_{i}$-the variables associated to the other $n$ constraints- stand for the price vector. The constraints of the dual problem are

$$
\begin{aligned}
\pi^{b}+\sum_{i \in S} \pi_{i} & \geq v(S) \quad \forall S \subseteq N \\
\pi^{b}, \pi_{i} & \geq 0
\end{aligned}
$$

The set of solutions of ILP is the set of optimal feasible solutions (vertex points) of $\mathbf{L P}$ because of its special structure. Notice that if we remove the redundant constraints of $\mathbf{L P}$ we are left with the constraint whose coefficients are equal to 1 and the non-negativity conditions on variables $S$, for all $S \subseteq$ $N$. It is well known that the solutions for such a problem are integer: the variable corresponding to the maximum coefficient in the objective function is set to 1 and the remaining variables are set to 0 . Hence, in our case, an integer solution always exists and it is the consumption set $\widetilde{S} \subseteq N$ such that $\widetilde{S} \in \arg \max _{S \subseteq N} v(S)$.

Moreover, by the fundamental duality theorem (see Dantzig, 1974, p.125), if the primal problem has an optimal feasible solution, so does its dual problem and the two optimal value functions are the same. Also notice that the set of solutions is a convex polyhedron. Denote this set by $\operatorname{sol}($.$) .$

Interpreting variables $\pi_{i}$ of the dual problem as firms prices, let us define $\Pi=\left\{\left\{\pi_{i}\right\}_{i \in N} \in R^{n} \mid \exists \pi^{b}\right.$ such that $\left(\pi^{b},\left\{\pi_{i}\right\}_{i \in N}\right) \in \operatorname{sol}(\mathbf{D L P})$, and there is no other $\left(\pi^{\prime b},\left\{\pi_{i}^{\prime}\right\}_{i \in N}\right) \in \operatorname{sol}(\mathbf{D L P})$, such that $\pi_{i}^{\prime} \geq \pi_{i}$, for all $i$ and $\exists j$ with $\left.\pi_{j}^{\prime}>\pi_{j}\right\}$

as the Pareto frontier of the projection of set $\operatorname{sol}(\mathbf{D L P})$ on coordinates $\left\{\pi_{i}\right\}_{i \in N}$. We will see below that $\Pi$ will provide the Nash equilibrium prices for our economy. Furthermore, the projection of a convex polyhedron is a convex polyhedron as well and then the set $\Pi$ is its Pareto frontier and can be expressed as the convex combination of adjacent vertices. A way to obtain some of these vertices is to consider, among all solutions of the dual problem, 
those maximizing $\sum_{i \in N} \pi_{i}$. More precisely, let us define the restricted dual problem, RDLP,

$$
\begin{array}{ll}
\operatorname{Max} & \sum_{i \in N} \pi_{i} \\
\text { s.t. } & \pi^{b}+\sum_{i \in S} \pi_{i} \geq v(S) \quad \forall S \subseteq N \\
& \pi^{b}+\sum_{i \in N} \pi_{i}=V(N) \\
& \pi^{b}, \pi_{i} \geq 0
\end{array}
$$

Obviously, $\operatorname{sol}(\mathbf{R D L P}) \subseteq \operatorname{sol}(\mathbf{D L P})$.

To generate all the frontier $\Pi$ we define a family of problems which take into account the lexicographic order of the solutions of DLP. To this end, let $\mu$ be an ordered partition of $N$ in the sense that the order of the elements in the partition is relevant. Thus, $\mu$ and $\mu^{\prime}$ can give rise to the same partition, but with a different order in their elements. Let $\Gamma$ denote the set of all the ordered partitions. Write $\mu=\left\{N_{1}, N_{2}, \ldots, N_{L}\right\} \in \Gamma$ to mean that under $\mu$ the first element of the partition is $N_{1}$, the second in $N_{2}$ and the last one in $N_{L}$. Note that $L$ can differ from one partition to another.

According to the partition-approach, define the dual problem, $\mu$-DLP, as

$$
\begin{array}{ll}
\operatorname{Max} & \sum_{l=1}^{L}\left(\sum_{i \in N_{l}} \pi_{i}\right) 10^{d(L-l)} \\
\text { s.t. } & \pi^{b}+\sum_{i \in S} \pi_{i} \geq v(S) \quad \forall S \subseteq N \\
& \pi^{b}+\sum_{i \in N} \pi_{i}=V(N) \\
& \pi^{b}, \pi_{i} \geq 0
\end{array}
$$

where $d$ is an integer ${ }^{2}$ such that $n \cdot v(S)<10^{d}$ for all $S \subseteq N$. Note that the dual problem for the trivial partition $\mu=\{N\}$, is defined by RDLP. Let $F(\mu, \pi)=\sum_{l=1}^{L}\left(\sum_{i \in N_{l}} \pi_{i}\right) 10^{d(L-l)}$.

\footnotetext{
${ }^{2}$ The general condition is $d$ to be a sufficiently high integer.
} 
The partition formulation does not change the constraints but makes the objective function vary. The objective function is an integer for which each set of $d$ (consecutive) digits are determined by $\sum_{i \in N_{l}} \pi_{i}$. Thus, the first $d$ digits are occupied by $\sum_{i \in N_{1}} \pi_{i}$, the second $d$ digits by $\sum_{i \in N_{2}} \pi_{i}$ and so on, and, finally, the last $d$ digits by $\sum_{i \in N_{L}} \pi_{i}$. In this way, $\operatorname{sol}(\mu-\mathbf{D L P}) \subseteq$ $\operatorname{sol}(\mathbf{D L P})$ so that, a solution in $\mu$-DLP gives one of the most preferred price vectors by firms in $N_{1}$; it gives one of the most preferred price vectors by the set of firms in $N_{2}$, among those most preferred by firms in $N_{1}$; and so on.

EXAMPLE 4 (continuation): Let us solve $\mu$-DLP for different partitions $\mu \in \Gamma$. First notice that $n \cdot v(S)=3 \cdot 10<10^{2}$, thus we fix the parameter $d=2$.

Let $\mu=\{\{1\},\{2\},\{3\}\}$. The solution of $\mu$-DLP is the one which solves,

$$
\begin{aligned}
\operatorname{Max} F(\mu, \pi)= & \operatorname{Max}\left\{\pi_{1} \cdot 10^{4}+\pi_{2} \cdot 10^{2}+\pi_{3} \cdot 10^{0}\right\} \\
& \text { subjet to the above constraints }\left(^{*}\right)
\end{aligned}
$$

and gives the highest possible payoff $\left(\pi_{1}=5\right)$ to firm 1 . Then, among all the solutions of DLP with $\pi_{1}=5$, the one which gives firm 2 the biggest payoff $\left(\pi_{2}=0\right)$ is selected and finally firm 3 receives $\pi_{3}=0$.

However, if $\mu^{\prime}=\{\{3\},\{2\},\{1\}\}$ then the solution of the corresponding $\mu^{\prime}$-DLP gives firm 3 the biggest possible payoff, $\pi_{3}=4$; then, among all the solutions of DLP with $\pi_{3}=4$, the one which gives firm 2 the biggest one, $\pi_{2}=0$ is selected and finally the payoff to firm 1 is $\pi_{1}=1$. Thus, the solution of $\mu^{\prime}$-DLP is $\pi_{1}=1, \pi_{2}=0, \pi_{3}=4$.

Finally, when $\mu^{\prime \prime}=\{N\}$, then $\operatorname{sol}\left(\mu^{\prime \prime}-\mathbf{D L P}\right)=\operatorname{sol}(\mathbf{R D L P})$, and $\pi_{1}=$ $3, \pi_{2}=\pi_{3}=2$ is the solution which maximizes the objective function $F(N, \pi)=\operatorname{Max}\left\{\pi_{1}+\pi_{2}+\pi_{3}\right\}$.

As we can see all these solutions belong to the $S P E$-price vector set of example 4.

The next Lemma shows that the dual solutions $\left\{\pi_{i}\right\}_{i \in N}$ achieved by different partitions are in the Pareto frontier $\Pi$.

Lemma 1 Let $\mu, \mu^{\prime} \in \Gamma$, let $\left(\pi^{b}, \pi\right) \in \operatorname{sol}(\mu$-DLP $)$ and let $\left(\pi^{\prime b}, \pi^{\prime}\right) \in \operatorname{sol}\left(\mu^{\prime}-\right.$ DLP). Then both $\pi$ and $\pi^{\prime}$ belong to $\Pi$. 
Proof: Suppose that $\pi_{i} \leq \pi_{i}^{\prime}$ for all $i \in N$ and $\pi_{k}<\pi_{k}^{\prime}$, for some $k \in N$. Let $\mu=\left\{N_{1}, N_{2}, \ldots, N_{L}\right\}$ then,

$$
\begin{aligned}
F(\mu, \pi) & =\sum_{l=1}^{L}\left(\sum_{i \in N_{l}} \pi_{i}\right) 10^{d(L-l)} \\
& <\sum_{l=1}^{L}\left(\sum_{i \in N_{l}} \pi_{i}^{\prime}\right) 10^{d(L-l)}=F\left(\mu, \pi^{\prime}\right)
\end{aligned}
$$

which implies that $\left(\pi^{b}, \pi\right)$ is not a solution of $\mu$-DLP, a contradiction.

Define the binary relation to be coarser than in set $\Gamma$ as follows. Given $\mu, \mu^{\prime} \in \Gamma$, we say that $\mu^{\prime}=\left\{N_{1}^{\prime}, \ldots, N_{L}^{\prime}\right\}$ is coarser than $\mu=\left\{N_{1}, \ldots, N_{M}\right\}$ if

$$
\begin{aligned}
N_{1}^{\prime} & =N_{1} \cup N_{2} \cup \ldots \cup N_{n_{1}} \\
N_{2}^{\prime} & =N_{n_{1}+1} \cup \ldots \cup N_{n_{2}} \\
\vdots & =\vdots \\
N_{L}^{\prime} & =N_{n_{L-1}} \cup \ldots \cup N_{M}
\end{aligned}
$$

Clearly this binary relation is reflexive, anti-symmetric and transitive so that it induces a partial order relation, with all the maximal chains ending in $N$ and starting in any of the total partitions of $N$.

Coarser partitions have more degrees of freedom and hence the sum of dual solutions $\left\{\pi_{i}\right\}_{i \in N}$ is bigger. This is proven in the following Lemma.

Lemma 2 Let $\mu, \mu^{\prime} \in \Gamma$, with $\mu$ coarser than $\mu^{\prime}$. Then $\pi^{b} \leq \pi^{\prime b}$, for all $\left(\pi^{b}, \pi\right) \in \operatorname{sol}(\mu-\mathbf{D L P})$ and $\left(\pi^{\prime b}, \pi^{\prime}\right) \in \operatorname{sol}\left(\mu^{\prime}\right.$-DLP $)$.

Proof: It suffices to prove it for two consecutive partitions of a maximal chain, $\mu=\left\{N_{1}, \ldots, N_{l}, \ldots, N_{L}\right\}, \mu^{\prime}=\left\{N_{1}, \ldots, N_{l_{1}}, N_{l_{2}}, \ldots, N_{L}\right\}$, where $N_{l}=$ $N_{l_{1}} \cup N_{l_{2}}$.

Clearly, $\sum_{i \in N_{k}} \pi_{i}=\sum_{i \in N_{k}} \pi_{i}^{\prime}$ for $k=1, \ldots, l-1$. Moreover, $\sum_{i \in N_{l}} \pi_{i} \geq$ $\sum_{i \in N_{l_{i}}} \pi_{i}^{\prime}+\sum_{i \in N_{l_{2}}} \pi_{i}^{\prime}$ and $\sum_{N_{l+1} \cup \ldots \cup N_{L}} \pi_{i} \geq \sum_{N S_{l+1} \cup \ldots \cup N_{L}} \pi_{i}^{\prime}$.

Hence, $\sum_{i \in N} \pi_{i} \geq \sum_{i \in N} \pi_{i}^{\prime}$ which implies that $\pi^{b} \leq \pi^{\prime b}$. 


\section{Subgame Perfect Nash-equilibria via Lin- ear Programming}

\subsection{Existence}

Propositions 2 and 3 establish that the Cartesian product of solutions of LP and the associated $\mu$-DLP are $S P E$-outcomes. First, we start with a general property which states that the prices of non-active firms are set equal to marginal costs at any solution of LP and DLP problems.

Lemma 3 Let the pair $\left\{S,\left(\widetilde{\pi}^{b}, \widetilde{\pi}\right)\right\}$ be a solution of LP and DLP respectively. Then for all $j \in N \backslash \widetilde{S}, \widetilde{\pi}_{j}=0$.

Proof: If $j \in N \backslash \widetilde{S}$, then the constraint $\sum_{S \ni j} y(S) \leq 1$ of $\mathbf{L P}$ is strict. Hence, by the complementary slackness condition, the dual variable associated to this constraint, $\widetilde{\pi}_{j}$ must be zero. Obviously, this property holds for any solution of $\mu$-DLP.

The next Proposition gives an existence result similar to that of TUW(1997). It shows that any element of $\langle\operatorname{sol}(\mathbf{L P}) \times \operatorname{sol}(\mu-\mathbf{D L P})\rangle$ is a subgame perfect Nash-equilibrium outcome, i.e., $\operatorname{sol}(\mathbf{L P})$ give the equilibrium consumption set and $\operatorname{sol}(\mu$-DLP) an equilibrium price vector, for some partition $\mu$. Notice that, according to the above lemma, the non-active firms, set prices equal to zero. Its proof appears in the Appendix.

Proposition 2 Let $v$ be a value function, let $\widetilde{S} \in \operatorname{sol}(\mathbf{L P})$ and let $\left(\widetilde{\pi}^{b}, \widetilde{\pi}\right)$ be a solution of $\mu$-DLP, for some partition $\mu$. Then $(\widetilde{S}, \widetilde{\pi})$ is an SPE-outcome.

We may apply this Proposition to examples 2 and 4 above.

EXAMPLE 2 (continuation): The unique primal solution is $S=\{1,2\}$. Moreover, the unique solution of the restricted dual problem (i.e. $\mu=N$ ) is $\operatorname{sol}(\mathbf{R D L P})=\{(0.5,(0.5,0.5,0))\}$. Thus, by Proposition $2(\{1,2\},(0.5,0.5,0)) \in$ $S P E$-outcome set and the buyer gets a surplus of $\pi^{b}=v(\{1,2\})-p_{1}-p_{2}=$ 0.5 .

EXAMPLE 4 (continuation): In section 3 we offered the solutions of several $\mu$-DLP's associated to some partitions. Here, we offer a full list of them: 


\begin{tabular}{cc}
\hline Solution $\left(\widetilde{\pi}^{b}, \tilde{\pi}\right)$ & Partition $\mu$ \\
\hline$(5,(5,0,0))$ & $\{\{1\},\{2,3\}\},\{\{1\},\{2\},\{3\}\},\{\{1\},\{3\},\{2\}\}$ \\
$(3,(3,2,2))$ & $\{\{1,2\},\{3\}\},\{\{1,3\},\{2\}\},\{\{2,3\},\{1\}\},\{\{1,2,3\}\}$ \\
$(5,(1,4,0))$ & $\{\{2\},\{1,3\}\},\{\{2\},\{1\},\{3\}\},\{\{2\},\{3\},\{1\}\}$ \\
$(5,(1,0,4))$ & $\{\{3\},\{1,2\}\},\{\{3\},\{1\},\{2\}\},\{\{3\},\{2\},\{1\}\}$ \\
\hline
\end{tabular}

Thus, $(N,(5,0,0)),(N,(3,2,2)),(N,(1,4,0))$ and $(N,(1,0,4))$ are $S P E$ outcomes. Notice that the buyer surplus in the second of them, $\widetilde{\pi}^{b}=v(N)-$ $p_{1}-p_{2}-p_{3}=3$, is lower than the one corresponding to the other $S P E$ outcomes, where $\widetilde{\pi}^{b}=5$ in all of them.

By the above Proposition, the solutions of $\mu$-DLP are the $S P E$-price vectors in which the non-active firms set prices equal to zero. However, as examples 1 and 3 show, there are $S P E$-price vectors in which these firms may set positive prices: in example $1,(\{1,3\},(0,1,1))$ is an $S P E$-outcome and the non-producing firm 2 sets a price equal to 1 . This is in clear contrast with example 2 , where the unique $S P E$-outcome, $(\{1,2\},(0.5,0.5,0))$, is such that the non-producing firm cannot set a positive price, given that, say firm 1 would increase the price of its product.

The next Proposition shows that when producing firms extract the entire buyer surplus, i.e., $\pi^{b}=0$, then the non-producing ones can price arbitrarily, thus extending the previous existence proof. Notice that in this case $\operatorname{sol}(\mathbf{R D L P})=\operatorname{sol}(\mathbf{D L P})$. Given $S \subseteq N$ and $p \in R_{+}^{n}$, write $p=\left(p_{S}, p_{N \backslash S}\right)$, then we can state the following:

Proposition 3 Let $v$ be a value function, let $\widetilde{S} \in \operatorname{sol}(\mathbf{L P})$ and $(0, \widetilde{\pi}) \in$ $\operatorname{sol}(\mathbf{R D L P})$, then $\left(\widetilde{S},\left(\widetilde{\pi}_{\widetilde{S}}, \pi_{N \backslash \widetilde{S}}\right)\right) \in S P E$-outcome set, for all $\pi_{N \backslash \widetilde{S}} \in R_{+}^{|N \backslash \widetilde{S}|}$.

Proof: See Appendix.

We show next the efficiency of any equilibrium consumption set. Given $S \subseteq N$ and $p \in R_{+}^{n}$, recall that $p^{S}=\left(p_{S}, 0\right)$ means that the non-active firms set prices equal to zero.

Lemma $4 \widetilde{S}$ is an $S P E$-consumption set if and only if $\widetilde{S} \in \arg \max _{S \subseteq N} v(S)$.

Proof: Let $\widetilde{S} \in \arg \max _{S \subseteq N} v(S)$, then $\widetilde{S} \in \operatorname{sol}(\mathbf{L P})$. We also know that $\operatorname{sol}(\mu$-DLP $) \neq \emptyset$, thus let $\left(\widetilde{\pi}^{b}, \tilde{\pi}\right) \in \operatorname{sol}(\mu$-DLP $)$. By Proposition 2, these solutions give an $S P E$-outcome, where $\widetilde{S}$ is the $S P E$-consumption set. 
Now, let us check that if $(\widetilde{S}, \widetilde{p})$ is an $S P E$-outcome, then $\widetilde{S} \in \arg \max _{S \subseteq N} v(S)$. If $(\widetilde{S}, \widetilde{p}) \in S P E$-outcome set, then $\left(\widetilde{S}, p^{\widetilde{S}}\right)$ is also an $S P E$ by C3 of Proposition 1 . Then by $\mathrm{C} 1$, for all $T \subseteq N, v(\widetilde{S})-\sum_{k \in \widetilde{S}} p_{k}^{\widetilde{S}} \geq v(T)-\sum_{k \in T} p_{k}^{\widetilde{S}}$. Thus, $v(\widetilde{S})-v(T) \geq \sum_{k \in \widetilde{S} \backslash T} p_{k} \geq 0$ and then $v(S) \geq v(T)$.

When $\pi^{b}=0$, then the proof runs similarly by applying Proposition 3 and the above reasoning.

Corollary 1 If $(S, p) \in S P E$-outcome set, then $S$ maximizes the social surplus.

Proof: If $(S, p) \in S P E$, then $S$ maximizes the sum of the buyer surplus and firms' profits since,

$$
V(N)=v(S)=\left(v(S)-\sum_{k \in S} p_{k}\right)+\sum_{k \in S} p_{k}
$$

\subsection{Characterization of the $S P E$-price set by Linear Programming duality}

In this subsection we offer the central result of the paper: the characterization of the $S P E$-price vectors of the economy $G(n+1, v)$. When the buyer surplus is positive and the non-active firms set their prices equal to marginal costs, the set of $S P E$-price vectors is set $\Pi$, i.e., the non-Pareto dominated convex hull of solutions of $\mu$-DLP for all $\mu \in \Gamma$. When $\pi^{b}=0$, this convex hull characterizes the prices of the producing firms and the other firms' prices are arbitrary.

Let $(S, p)$ and $\left(S, p^{S}\right)$ be two $S P E$-outcomes, where $p^{S}=\left(p_{S}, 0\right)$. Then clearly, firms and the buyer obtain the same payoffs under such outcomes: the two equilibria are payoff-equivalent. Thus, any pair $\left(S, p^{S}\right)$ allows us to identify its payoff equivalence class. For any set of payoff equivalent $S P E-$ outcomes, we are only considering $\left(S, p^{S}\right)$ as the representative outcome of this equivalence class.

Before proving the main result we offer some properties of the $S P E$ outcome set. The first one says that any product not belonging to all the 
$S P E$-consumption bundles is priced at zero. The intuition behind is that if a product does not belong to all the optimal consumption sets it can be easily substituted for any other product and then it is priced at marginal cost. Furthermore, given an $S P E$-price vector $p$ supporting an equilibrium consumption bundle $\widetilde{S}$ and given any other optimal consumption $S$, the transformation of $p$, denoted by $p^{S}$, where prices of products outside $S$ are equal to zero, is also an $S P E$-price vector supporting $S$.

These two facts summarize the remarkable property that given an $S P E$ price vector $p$, then any optimal consumption bundle $S$ is always supported by $p^{S}$. In other words, there is not a bijective mapping between equilibrium price vectors and equilibrium consumption bundles and this is why any element of the Cartesian product $\langle\operatorname{sol}(\mathbf{L P}) \times \operatorname{sol}(\mu-\mathbf{D L P})\rangle$ is a Nash-equilibrium outcome as shown in Proposition 2 above.

Lemma 5 (i) Let $D=\left\{k \in N \mid k \in S\right.$ for all $\left.S \in \arg \max _{K \subseteq N} v(K)\right\}$. If $i \notin D$, then $p_{i}=0$, for all $p \in S P E$-price set.

(ii) If $(\widetilde{S}, p)$ is an $S P E$-outcome and $S \in \arg \max _{K \subseteq N} v(K)$ then $\left(S, p^{S}\right) \in$ $S P E$-outcome set, where $p^{S}=\left(p_{S}, 0\right)$ is defined from $p$.

Proof: (i) We show that if $(S, p) \in S P E$-outcome set, then $p_{i}=0$ for all $i \in S \backslash D$. If $S \backslash D=\emptyset$ then the result trivially holds. Let $i \in S \backslash D$, then there exists $S^{i} \in \arg \max _{K \subseteq N} v(K)$ such that $i \notin S^{i}$. If $(S, p) \in S P E$ outcome set, then $\left(S, p^{S}\right) \in S P E$-outcome set and $S \in \arg \max _{K \subseteq N} v(K)$ (Lemma 4). By C1, $v(S)-\sum_{k \in S} p_{k}^{S} \geq v\left(S^{i}\right)-\sum_{k \in S^{i}} p_{k}^{S}$ which implies that $0 \geq \sum_{k \in S} p_{k}^{S}-\sum_{k \in S^{i}} p_{k}^{S}=\sum_{k \in S \backslash S^{i}} p_{k}^{S}$. Thus $p_{k}^{S}=0$ for all $k \in S \backslash S^{i}$ (and $i$ $\left.\in S \backslash S^{i}\right)$.

Moreover, if $(S, p) \in S P E$-outcome set, then $p^{S}=p^{D},\left(S, p^{D}\right) \in S P E$ outcome set and the buyer surplus is the same, i.e., $v(S)-\sum_{k \in S} p_{k}=v(S)-$ $\sum_{k \in S} p_{k}^{D}$.

(ii) It suffices to prove that $\left(S, p^{\widetilde{S}}\right) \in S P E$-outcome set, given that $p^{S}=$ $p^{\widetilde{S}}=p^{D}$, i.e., that $v(\widetilde{S})-\sum_{k \in \widetilde{S}} p_{k}^{\widetilde{S}}=v(S)-\sum_{k \in S} p_{k}^{\widetilde{S}}$.

By C1,

$$
v(\widetilde{S})-\sum_{k \in \widetilde{S}} p_{k}^{\widetilde{S}} \geq v(S)-\sum_{k \in S} p_{k}^{\widetilde{S}}
$$

Moreover, 


$$
v(S)-\sum_{k \in S} p_{k}^{\widetilde{S}}=v(\widetilde{S})-\sum_{k \in S} p_{k}^{\widetilde{S}} \geq v(\widetilde{S})-\sum_{k \in \widetilde{S}} p_{k}^{\tilde{S}}
$$

where the equality holds since by Lemma $4, \widetilde{S} \in \arg \max _{K \subseteq N} v(K)$ and the inequality holds since $p_{k}^{\widetilde{S}}=0$ if $k \in S \backslash \widetilde{S}$. set.

Hence $v(S)-\sum_{k \in S} p_{k}^{\widetilde{S}}=v(\widetilde{S})-\sum_{k \in \widetilde{S}} p_{k}^{\widetilde{S}}$ and $\left(S, p^{\widetilde{S}}\right) \in S P E$-outcome

The next result states that Pareto-dominated prices can never be $S P E$ prices.

Lemma 6 Let $p \in R_{+}^{n}$, if there exists $(\widetilde{S}, \widetilde{p}) \in S P E$-outcome set such that $\widetilde{p}^{\widetilde{S}} \geq p^{\widetilde{S}}$ and $\widetilde{p}_{j}>p_{j}$ for some $j \in \widetilde{S}$, then $p$ is not an SPE-price vector.

Proof: If $p$ is an $S P E$-price vector then $p^{\widetilde{S}}$ is also an $S P E$-price vector, thus we only have to prove that $p^{\widetilde{S}}$ is not an $S P E$-price vector.

$(\widetilde{S}, \widetilde{p}) \in S P E$-outcome set, hence $(\widetilde{S}, \widetilde{p} \widetilde{S}) \in S P E$-outcome set. Let $S_{j} \subseteq$ $N \backslash\{j\}$, by $\mathrm{C} 1$

$$
v(\widetilde{S})-\sum_{k \in \widetilde{S}} \widetilde{p}_{k}^{\widetilde{S}} \geq v\left(S_{j}\right)-\sum_{k \in S_{j}} \widetilde{p}_{k}^{\widetilde{S}}
$$

and since $j \notin S_{j}$

$$
\begin{aligned}
\sum_{k \in \widetilde{S}}\left(\widetilde{p}_{k}^{\widetilde{S}}-p_{k}^{\widetilde{S}}\right) & =\sum_{k \in \widetilde{S} \backslash S_{j}}\left(\widetilde{p}_{k}^{\widetilde{S}}-p_{k}^{\widetilde{S}}\right)+\sum_{k \in \widetilde{S} \cap S_{j}}\left(\widetilde{p}_{k}^{\widetilde{S}}-p_{k}^{\widetilde{S}}\right) \\
& >\sum_{k \in \widetilde{S} \cap S_{j}}\left(\widetilde{p}_{k}^{\widetilde{S}}-p_{k}^{\widetilde{S}}\right)=\sum_{k \in S_{j}}\left(\widetilde{p}_{k}^{\widetilde{S}}-p_{k}^{\widetilde{S}}\right)
\end{aligned}
$$

Thus,

$$
\begin{aligned}
v(\widetilde{S})-\sum_{k \in \widetilde{S}} p_{k}^{\widetilde{S}} & =v(\widetilde{S})-\sum_{k \in \widetilde{S}} \widetilde{p}_{k}^{\widetilde{S}}+\sum_{k \in \widetilde{S}}\left(\widetilde{p}_{k}^{\widetilde{S}}-p_{k}^{\widetilde{S}}\right)> \\
& >v\left(S_{j}\right)-\sum_{k \in S_{j}} \widetilde{p}_{k}^{\widetilde{S}}+\sum_{k \in S_{j}}\left(\widetilde{p}_{k}^{\widetilde{S}}-p_{k}^{\widetilde{S}}\right)= \\
& =v\left(S_{j}\right)-\sum_{k \in S_{j}} p_{k}^{\widetilde{S}}
\end{aligned}
$$


for all $S_{j} \subseteq N \backslash\{j\}$ and hence C2 never holds for $p^{\widetilde{S}}$.

Notice first that any $S P E$-price vector where non-active firms set prices equal to marginal costs is a solution of DLP, since it can be easily checked that $S P E$-price vectors satisfying $(\mathrm{C} 1)-(\mathrm{C} 3)$ verify all the constraints of the linear programming problem. Then, since by Lemma 6 , if $\widetilde{p} \in S P E$-price vector, then there does not exist any other $\left(\pi^{b}, \pi^{\widetilde{S}}\right) \in \operatorname{sol}(\mathbf{D L P})$ such that $\pi^{\widetilde{S}}$ weakly Pareto-dominates $\widetilde{\pi}^{S}$, the solutions of $\mu$-DLP are equilibrium prices (see Lemma 1).

Proposition 4 Let $v$ be a value function. $\left(\widetilde{S}, \widetilde{p}^{\widetilde{S}}\right) \in S P E$-outcome set if and only if

i) $\widetilde{S} \in \operatorname{sol}(\mathbf{L P})$,

ii) $\left(\widetilde{\pi}^{b}, \widetilde{\pi}^{\widetilde{S}}\right) \in \Pi$, i.e., it is a convex combination of solutions of $\mu$-DLP problems, where $\widetilde{\pi}^{b}=v(\widetilde{S})-\sum_{k \in \widetilde{S}} \widetilde{p}_{k}^{\widetilde{S}}$, and $\widetilde{\pi}^{\widetilde{S}}=\widetilde{p}^{\widetilde{S}}$.

Proof: See Appendix.

Corollary 2 Let $v$ be a value function, let $\widetilde{S} \in \operatorname{sol}(\mathbf{L P})$ and let $\left(\widetilde{\pi}^{b}, \widetilde{\pi}\right)$ be a solution of RDLP. Then $(\widetilde{S}, \widetilde{\pi})$ is the SPE-outcome which gives the lowest surplus to the buyer.

Proof: By Lemma 2, where $\mu=\{N\}$ is the coarsest partition, $(\widetilde{S}, \widetilde{\pi})$ is the $S P E$-outcome which gives the lowest surplus to the buyer.

The next results relates $S P E$-outcomes with both $\operatorname{core}(G)$ and $W E$ outcomes. To this end let $W E$ be the set of Walrasian equilibrium outcomes. Recall that $(S, p)$ is a $W E$-outcome iff $p \geq 0$ (or marginal costs), and

(i) $v(S)-\sum_{k \in S} p_{k} \geq v(T)-\sum_{k \in T} p_{k}$ for all $T \subseteq N$

(ii) If $i \notin S$ then $p_{i}=0$.

The next proposition shows that $\operatorname{core}(G)$ coincides with the solutions of the dual linear problem and that it is completely priced by the set of $W E$ prices.

Proposition 5 Let $v$ be a value function which defines the economy $G(n+$ $1, v, c=0)$ and let $T \in \arg \max _{S \subseteq N}\{v(S)\}$. Then,

i) $\operatorname{sol}(\mathbf{D L P})=T-\operatorname{core}(G)$

ii) $\left(p^{b}, p\right) \in T$-core $(G)$ if and only if $(T, p) \in W E$ and $p^{b}=v(T)-\sum_{k \in T} p_{k}$ 
Proof: First, let us prove that $\operatorname{sol}(\mathbf{D L P})=\operatorname{core}(G)$. If $\left(p^{b}, p\right) \in \operatorname{sol}(\mathbf{D L P})$, then by the constraints of the dual problem ,

$$
p^{b}+\sum_{S} p_{i}=p^{b}+\sum_{S \cap T} p_{i} \geq v(S)
$$

for all $S \subseteq N$, and by the fundamental duality theorem

$$
V(N)=p^{b}+\sum_{N} p_{i}=p^{b}+\sum_{T} p_{i}=v(T)
$$

Hence $\left(p^{b}, p\right) \in T$-core $(G)$.

On the other hand, let $\left(p^{b}, p\right) \in T$-core $(G)$, then $p_{k}=0$ for all $k \notin T$ and by condition ii) of the definition of the core,

$$
p^{b}+\sum_{S \cap T} p_{i}=p^{b}+\sum_{S} p_{i} \geq v(S)
$$

for all $S \subseteq N$, and the constraints of DLP are satisfied. Moreover, by condition i) of the definition of the core $p^{b}+\sum_{T} p_{i}=p^{b}+\sum_{N} p_{i}=v(T)$, thus $\left(p^{b}, p\right) \in \operatorname{sol}(\mathbf{D L P})$.

Now, let us prove ii). By the first welfare theorem if $(T, p) \in W E$-outcome set, and $p^{b}=v(T)-\sum_{k \in T} p_{k}$, then $\left(p^{b}, p\right) \in T$-core $(G)$, thus we only have to show that if $\left(p^{b}, p\right) \in T$-core $(G)$. Then $(T, p) \in W E$-outcome set. Let $\left(p^{b}, p\right) \in T$-core $(G)$ then $p_{k}=0$ for all $k \notin T$ and condition (ii) of the $W E$ characterization holds. Besides, $p^{b}=v(T)-\sum_{k \in T} p_{k}$ and given $S \subseteq N$, $p^{b}+\sum_{k \in S} p_{k} \geq v(S)$. Thus, $v(T)-\sum_{k \in T} p_{k}+\sum_{k \in S} p_{k} \geq v(S)$, which implies condition (i) of the $W E$ characterization.

By the above proposition we have,

Corollary 3 The set of SPE-prices, where the non-active firms set prices equal to zero, jointly with the associated buyer surplus is a subset of $\operatorname{core}(G)$. Then, these SPE-prices are a subset of the WE-prices.

Furthermore,

Lemma 7 Let $(S, p) \in W E$-outcome set such that there exists no $\left(S^{\prime}, p^{\prime}\right) \in$ $W E$ with $p_{i}^{\prime} \geq p_{i}$ for all $i \in N$ and $p_{k}^{\prime}>p_{k}$ for some $k \in N$. Then $(S, p) \in S P E$-outcome set. 
Proof: Let $(S, p)$ be a $W E$-outcome which satisfies the above assumptions. We prove that $(S, p)$ verifies $\mathrm{C} 1$ to $\mathrm{C} 3$. By condition (i) of the definition of $W E, \mathrm{C} 1$ is satisfied. C3 holds given that $p_{i}=0$ for $i \notin S$. Finally, suppose that C2 is not verified, i.e., there exists $j \in S$ such that for all $S^{\prime} \subseteq N \backslash\{j\}$

$$
v(S)-\sum_{k \in S} p_{k}>v\left(S^{\prime}\right)-\sum_{k \in S^{\prime}} p_{k}
$$

Let $p^{\prime}$ be a price vector such that $p_{i}^{\prime}=p_{i}$ for all $i \in N \backslash\{j\}$ and $p_{j}^{\prime}=p_{j}+\epsilon$, where $\epsilon<\max _{S^{\prime} \subseteq N \backslash\{j\}}\left\{v(S)-\sum_{k \in S} p_{k}-v\left(S^{\prime}\right)-\sum_{k \in S^{\prime}} p_{k}\right\}$. Then we have that for all $T \subseteq N$,

$$
v(S)-\sum_{k \in S} p_{k}^{\prime}>v(T)-\sum_{k \in T} p_{k}^{\prime}
$$

and $p_{i}^{\prime}=0$ for all $i \notin S$. Thus, $\left(S, p^{\prime}\right) \in W E$-outcome set and $p^{\prime}$ Pareto dominates $p$ which contradicts the assumption. Then $\mathrm{C} 2$ is also satisfied.

\section{Some particular value functions}

In this section we characterize the $S P E$-outcomes for concave and $k$-convex monotonic value functions and identify some explicit $S P E$-price vectors. We will show that when $v$ is convex, symmetric $k$-convex or 1-convex, some central solution concepts in cooperative game theory, such as the Shapley value and the nucleolus are (subgame perfect) $S P E$-prices of our proposed non-cooperative game. When $v$ is concave, the $S P E$-prices are the marginal values of the products. Moreover, in this case the $S P E$-price set is a singleton and, in turn, coincides with the maximal Walrasian price. We begin with some definitions.

Definition $3 v$ is monotonic if and only if $v(S) \leq v(T)$ whenever $S \subseteq T \subseteq$ $N$.

Monotonicity of $v$ implies that the buyer's willingness to pay increases for larger consumption sets. 
Definition 4 (1) $v$ is convex if and only if

$$
v(S+i)-v(S) \leq v(T+i)-v(T)
$$

whenever $S \subseteq T \subseteq N \backslash i$, and

(2) $v$ is concave if and only if

$$
v(S+i)-v(S) \geq v(T+i)-v(T)
$$

The convexity of $v$ reflects a kind of complementarity among products: the amount a buyer is willing to pay for an additional good increases with the number of products he/she is consuming. Alternatively, the concavity of $v$ implies that the amount a buyer is willing to pay for a good decreases with the number of products he/she is consuming ${ }^{3}$.

Lemma 8 If $v$ is a monotonic value function then there exists an NEoutcome of the form $(N, p)$. Furthermore, if $v$ is strictly monotonic then $N$ is the unique SPE-consumption set.

Proof: It suffices to show that $N \in \operatorname{sol}(\mathbf{L P})$, but this trivially holds given that if $v$ is monotonic then $v(N) \geq v(S)$ for all $S \subseteq N$. Hence, $N \in \arg \max _{S \subseteq N} v(S)$ and by Lemma $4, N$ is an $S P E$-consumption set. If the value function is strictly monotonic, then $N$ is the only element in $\arg \max _{S \subseteq N} v(S)$.

\subsection{Concave value functions}

Concavity of $v$ reflects a kind of substitution among products so that market competition will permit the buyer to retain some surplus. Let us define,

Definition 5 Let $v$ be monotonic, the marginal contribution of any product $i \in N$ is defined as

$$
c^{*}(i)=v(N)-v(N \backslash i)
$$

\footnotetext{
${ }^{3}$ Notice that $v$ is convex if and only if

$$
v(S \cup T)+v(S \cap T) \geq v(S)+v(T)
$$

for any two subsets $S$ and $T$ of $N$, i.e., if $v$ is supermodular. Similarly, $v$ is concave if and only if $v$ is submodular.
} 
and the marginal contribution of any subset of products $S \subseteq N$ is

$$
c^{*}(S)=v(N)-v(N \backslash S)
$$

Equivalently, the gap function $g: 2^{N} \longrightarrow R$ is given by

$$
g(S)=\sum_{i \in S} c^{*}(i)-v(S), \text { for all } S \subseteq N
$$

Notice that by $\mathrm{C} 1$, if $(N, \widetilde{p})$ is an NE-outcome then $v(N)-\sum_{k \in N} \widetilde{p}_{k} \geq$ $v(N \backslash i)-\sum_{k \in N \backslash i} \widetilde{p}_{k}$, for any $i \in N$. Thus $c^{*}(i)=v(N)-n(N \backslash i) \geq \widetilde{p}_{i}$. Hence, if $g(N) \leq 0$ (or equivalently $v(N) \geq \sum_{i \in N} c^{*}(i)$ ) and every firm sets its price equal to its marginal contribution, then the buyer surplus is still positive.

Following Shapley (1962), we say that "products are substitutes" if for all $S \subseteq N$

$$
c^{*}(S) \geq \sum_{i \in S} c^{*}(i)
$$

The 'product substitution' property, PS, implies that the marginal contribution of a consumption set $S$ is bigger than the sum of those of the products in $S$, and we will see that this is implied by the concavity of $v$ (see Lemma 10). This property has been previously used in different settings by several authors such as KC (1982) and BO (2001), among others. The former have employed it to justify that workers are better off by forming a union rather than by bargaining individually with management, whereas the latter to show that when buyers are substitutes, then the core has the lattice property with respect to the buyers. In our economy, when property PS holds, the $S P E$-price vector consists of the marginal contributions of the products in the consumption set.

Proposition 6 If the value function is monotonic and PS holds, then $(\widetilde{S}, \widetilde{p}) \in$ $S P E$-outcome set if and only if

(i) $\widetilde{S} \in \operatorname{sol}(\mathbf{L P})$, with $v(\widetilde{S})=v(N)$

(ii) The equilibrium price of each product $i \in N$ is $\widetilde{p}_{i}=c^{*}(i)$, the marginal contribution of product $i$.

Proof: See Appendix.

We next prove that the above result is satisfied when $v$ is concave. 
Lemma 9 Let $v$ be a concave value function and let $c^{*}(i) \geq 0$ for all $i \in N$.

Then $v$ is monotonic.

Proof: Let $i \neq j, i, j \in N$. By concavity,

$$
v(N \backslash j)-v(N \backslash\{i, j\}) \geq v(N)-v(N \backslash i) \geq 0
$$

thus $v(N \backslash j) \geq v(N \backslash\{i, j\})$. In general, it can be proven by induction that

$$
v\left(N \backslash\left\{i_{1}, \ldots, i_{l}\right\}\right) \geq v\left(N \backslash\left\{i_{1}, \ldots, i_{l}, i_{l+1}\right\}\right),
$$

i.e., $v(S) \leq v(S+i)$.

Let $S \subseteq T \subseteq N$ and $T \backslash S=\left\{i_{1}, \ldots, i_{l}\right\}$. We have that $v(S) \leq v\left(S \cup\left\{i_{1}\right\}\right) \leq$ $\ldots \leq v\left(S \cup\left\{i_{1}, \ldots, i_{l-1}\right\}\right) \leq v(T)^{4}$.

Lemma 10 Let $v$ be a concave value function, then PS holds, i.e., for all $S \subseteq N$,

$$
c^{*}(S) \geq \sum_{i \in S} c^{*}(i)
$$

Proof: Let $S=\left\{i_{1}, \ldots, i_{l}\right\}$. Then,

$$
\begin{aligned}
c^{*}(S) & =v(N)-v(N \backslash S) \\
& =v(N)-v\left(N \backslash i_{1}\right)+\sum_{j=1}^{l-1}\left[v \left(N \backslash\left\{i_{1}, \ldots, i_{j}\right\}-v\left(N \backslash\left\{i_{1}, \ldots, i_{j+1}\right\}\right]\right.\right. \\
& \geq \sum_{j=1}^{l} v(N)-v\left(N \backslash i_{j}\right)=\sum_{i \in S} c^{*}(i)
\end{aligned}
$$

Now, by Lemmas 9-10 and Proposition 6, the following corollary is obtained.

Corollary 4 If the value function is concave and $c^{*}(i) \geq 0$ for all $i$, then the result of Proposition 6 applies.

\footnotetext{
${ }^{4} \mathrm{~A}$ monotonic function need not be either concave or convex. For instance, the value function, $v(1)=1, v(2)=2$ and $v(1,2)=x$, is monotonic if $x \geq 2$, but it is convex for $x=3.5$ while concave for $x=2.5$.
} 
Remark: By the previous corollary, when $v$ is lineal, i.e. $v(S)=\sum_{S} v(i)$ for all $S \subseteq N$, then $(\widetilde{S}, \widetilde{p}) \in S P E$-outcome set if and only if $v(\widetilde{S})=v(N)$ and $\widetilde{p}_{i}=v(i)$ for all $i \in N$. If $v($.$) is linear then it is concave and monotonic. Thus$ any efficient consumption set is such that $v(\widetilde{S})=v(N)$ and firm $i$ 's price is its marginal contribution, but $c^{*}(i)=v(N)-v(N \backslash i)=\sum_{N} v(i)-\sum_{N \backslash i} v(i)=$ $v(i)$.

Recall that by Proposition 5 the $S P E$-price vector set is a subset of $T$-core $(G)$. Hence, for monotonic value function satisfying product substitution $T$-core $(G)=\operatorname{core}(G)$ and the core has the lattice property (Shapley and Shubik, 1972). Let $\bar{p}^{b}$ and $p^{b}$ denote the highest and lowest buyer surplus, respectively, and similarly define $\bar{p}_{k}$ and $\underline{p}_{k}$ for each firm $k \in N$. The lattice property of $\operatorname{core}(G)$ states that vectors $\left(\bar{p}^{b},\left(\underline{p}_{k}\right)_{k \in N}\right)$ and $\left(\underline{p}^{b},\left(\bar{p}_{k}\right)_{k \in N}\right)$ are themselves in core $(G)$. Moreover, no two vectors in core $(G)$ are further apart than these two.

In our case firm $k$ 's marginal contribution is an upper bound of $p_{k}$ and $c^{*}(0)=v(N)-\sum_{i \in S} c^{*}(i)$ is a lower bound of the buyer's surplus, i.e., $p_{k} \leq$ $c^{*}(k)$ and $p^{b} \geq c^{*}(0)$, at any point in core $(G)$. Then $\bar{p}_{k}=c^{*}(k), \underline{p}_{k}=0$, $\underline{p}^{b}=c^{*}(0)$ and $\bar{p}^{b}=v(N)$, and by the above corollary, the most preferred point by all firms and the least preferred by the buyer in $\operatorname{core}(G)$, i.e. $\bar{C}^{*}=$ $\left(c^{*}(1), \ldots, c^{*}(n)\right)$ is implemented as the unique $S P E$-price vector.

The next result relates $S P E$-outcomes with $W E$-outcomes. When $v$ is monotonic and concave (or satisfies product substitution), the lattice property of core $(G)$ translates to the $W E$-price set. Thus, the maximal $W E$-price vector is implemented as the unique $S P E$-price vector. Let us summarize the above discussion.

Corollary 5 If $v$ is monotonic and concave (or PS holds), then the most preferred point by all firms in core $(G)$ is priced as the unique SPE-price vector of the economy $G$. Moreover, this SPE-price vector coincides with the maximal $W E$-price vector.

EXAMPLE 2 (continuation): Function $v$ satisfies condition PS. The unique subgame perfect Nash-equilibrium outcome is $(S, p)=(\{1,2\},(0.5,0.5,0))$, while the $W E$-outcome is, $\operatorname{sol}(\mathbf{L P})=\{1,2\}$ and $\operatorname{sol}(\mathbf{D L P})=\{(1.5-\alpha-$ $\beta, \alpha, \beta, 0) \mid 0 \leq \alpha \leq 0.5,0 \leq \beta \leq 0.5\}$. The maximal $W E$-price vector coincides with the unique $S P E$-price vector. 


\section{$5.2 k$-convex value functions}

In this section we characterize the set of $S P E$-price vectors for a wide family of value functions: $k$-convex value functions (see Driessen, 1986, 1988 and Driessen and Rafels, 1999). For these functions the set of SPE-price vectors is the convex hull of $n$ ! price vectors which are the corner solutions of RDLP. We offer the explicit value of these corner solutions.

It is not difficult to show that if $v$ is monotonic and $k$-convex, with $k \geq 1$, then $\operatorname{core}(v)$ is non-empty and it is a subset of $\operatorname{core}(G)$. In fact, $\operatorname{core}(v)$ is the set of solutions of RDLP. On the other hand, when $v$ is monotonic and the buyer surplus is zero, the $S P E$-price vector set is also the set of solutions of RDLP, thus coinciding with core $(v)$. Furthermore, if $v$ is either convex or symmetric $k$-convex, the Shapley value of $v$ is an $S P E$-price vector; while if $v$ is 1-convex, the nucleolus of $v$ is what is an equilibrium price vector.

Consider first that $v$ is convex, i.e., $k=n$. Then, the gap function $g(N)$ is nonnegative (or equivalently $v(N) \leq \sum_{i \in N} c^{*}(i)$ ) and firms cannot set prices equal to their marginal contributions. However, as next Proposition states, firms extract all the buyer surplus $\left(\pi^{b}=0\right)$ and the set of solutions of RDLP coincides with the set of $S P E$-price vectors.

Proposition 7 If the value function is convex then $(\widetilde{S}, \widetilde{\pi}) \in S P E$-outcome set if and only if $\widetilde{S} \in \operatorname{sol}(\mathbf{L P})$ and $\left(0, \widetilde{\pi}^{S}\right) \in \operatorname{sol}(\mathbf{R D L P})$.

Proof: See Appendix.

The convexity of function $v$ reflects complementarities among products and therefore it induces only weak market competition so that firms can extract the entire buyer surplus. Moreover, if $v$ is nonnegative and convex it is straightforward to prove that $v$ is monotonic as the next Lemma shows.

Lemma 11 Let $v$ be a convex value function with $v(i) \geq 0$ for all $i \in N$. Then $v$ is monotonic.

Proof: By convexity of $v$ and since $v(i) \geq 0$ for all $i \in N$, given $i, j, k \in N$,

$$
\begin{aligned}
v(i, j)-v(i) & \geq v(j)-v(\emptyset) \geq 0 \Longrightarrow v(i, j) \geq v(i) \geq 0 \\
v(i, j, k)-v(i, j) & \geq v(k)-v(\emptyset) \geq 0 \Longrightarrow v(i, j, k) \geq v(i, j) \geq 0
\end{aligned}
$$


Thus, $v(i, j, k) \geq v(i, j) \geq v(i) \geq 0$. Extending this argument for any set $S=\left\{i_{1}, i_{2}, \ldots, i_{s}\right\} \subseteq N$ it yields,

$$
v(S) \geq v\left(i_{1}, \ldots, i_{s-1}\right) \geq \ldots \geq v\left(i_{1}, i_{2}\right) \geq v\left(i_{i}\right) \geq 0
$$

for any order of the elements of $S$. Hence, given $T \subseteq S \subseteq N$, it is verified that $v(T) \leq v(S)$.

By Proposition 7, the set of subgame perfect Nash-equilibrium price vectors are the solutions of RDLP and then they are a subset of Walrasian equilibrium prices (see Proposition 5). Furthermore, the solutions of RDLP can be expressed as the convex hull of at most the $n$ ! corner solutions. Again, some definitions are needed.

Definition 6 Let $\Sigma$ be the set of permutations (orderings) of $N=\{1,2, \ldots, n\}$ and let $\sigma \in \Sigma$ be any of its elements. Let $P_{i}^{\sigma}$ be the set of firms which precede firm $i$ with respect to permutation $\sigma$, i.e., for all $i \in N$ and $\sigma \in \Sigma$,

$$
P_{i}^{\sigma}=\{j \in N \mid \sigma(j)<\sigma(i)\}
$$

Definition 7 (Shapley, 1971) The marginal contribution vector $x^{\sigma}(v) \in$ $R^{n}$ of $v$ with respect to ordering $\sigma$ is given by,

$$
x_{i}^{\sigma}(v)=v\left(P_{i}^{\sigma}+i\right)-v\left(P_{i}^{\sigma}\right), \text { for all } i \in N
$$

If $v$ is convex, then the marginal contribution vector $x^{\sigma}(v)$ is positive and each firm is given its marginal contribution with respect to the economy consisting of all its predecessors. Moreover $x^{\sigma}(v)$, for all $\sigma \in \Sigma$, are the corner solutions of RDLP. Then,

Proposition 8 Let $v$ be a convex value function, such that $v(i) \geq 0$ for all $i \in N$. The following three sets are the same.

(i) $\operatorname{Core}(v)$

(ii) SPE-price vector set

(iii) $\operatorname{conv}\left\{x^{\sigma}(v) \mid \sigma \in \Sigma\right\}$

Proof: (i) $\Longleftrightarrow$ (ii) is given by propositions 4 and 7 jointly with the fact that if the buyer surplus is zero, then the set of solutions of RDLP is the same than that of $\operatorname{core}(v) \subset \operatorname{Core}(G)$. 
(i) $\Longleftrightarrow($ iii) is show in Driessen (1993).

Moreover, when $v$ is convex, the centre of gravity of all solutions $x^{\sigma}(v)$, for all $\sigma \in \Sigma$ is in $\operatorname{Core}(v)$ and coincides with the Shapley value of $v$. The Shapley value can be interpreted as the expected marginal contribution of a firm to the value of each subset of products, where the distribution of subsets of products is such that any ordering of firms is equally likely.

Corollary 6 Let $v$ be a convex value function, such that $v(i) \geq 0$ for all $i \in N$. The Shapley value of $v, \phi$, is an SPE-price vector.

Proof: If $v$ is convex, then the Shapley value vector, $\phi$, is the average of all the corner points $x^{\sigma}(v)$, thus $\phi \in \operatorname{conv}\left\{x^{\sigma}(v) \mid \sigma \in \Sigma\right\}$. It is not difficult to calculate that,

$$
\phi_{i}=\frac{1}{n !} \sum_{\sigma \in \Sigma} x_{i}^{\sigma}=\sum_{S \subseteq N \backslash i} \frac{|S| !(n-|S|-1) !}{n !}[v(S+i)-v(S)] \quad \forall i=1, \ldots, n .
$$

Some of the above results for convex value functions can be extended to the family of $k$-convex value functions. The notion of $k$-convexity can be regarded as some kind of weak convexity. In fact, $n$-convexity agrees with convexity. As shall be shown below, under $k$-convexity, firms still extract all the consumer surplus, and an interpretation of $k$-convexity is as follows. Suppose that the formation of the market with $n$ firms (and hence $n$ products) is seen as a sequential process where firms enter one at a time until a market of $k-1$ firms is formed and subsequently, the remaining firms enter altogether. In view of this specific formation of the total market, $k$-convexity expresses that the amount a buyer is willing to pay for an additional good increases with the number of products (firms) she is consuming. So, the critical number $k$ is related to the size of the market and it is used to indicate that large markets, consisting of at least $k$ products (firms), yield lower profits for firms (in particular, their prices will be smaller than their marginal contributions), whereas small markets, consisting of at most $k-1$ products (firms) yield higher profits as compared with the value of the total market, $v(N)$. Formally,

Definition 8 Let $v$ be a value function and $k \in N$. We say that $v$ is $k$ convex if and only if $v$ satisfies the following four conditions: 
- $v(S+i)-v(S) \leq v(T+i)-v(T)$

(or in terms of the gap function: $g(S+i)-g(S) \geq g(T+i)-g(T)$ )

for all $i \in N$ and all $S \subset T \subseteq N \backslash i$ with $|T| \leq k-2$

- $v(N)-v(T) \geq \sum_{N \backslash(T+i)} c^{*}(k)+\max _{j \in T}\{v((T+i) \backslash j)-v(T \backslash j)\},(D \mathscr{2})$

(or equivalently given (D1): $g(S+i)-g(S) \geq g(N)-g(T)$ ),

for all $i \in N$ and all $T \subseteq N \backslash i$ with $T \neq \emptyset,|T|=k-1$

- $c^{*}(N \backslash S) \leq \sum_{N \backslash S} c^{*}(k),($ or $g(S) \leq g(N))$,

for all $S \subseteq N$ with $|S|=k-1$

- $c^{*}(N \backslash S) \geq \sum_{N \backslash S} c^{*}(k),($ or $g(S) \geq g(N))$,

for all $S \subseteq N$ with $|S| \geq k$

We develop an interpretation of conditions (D1) to (D4). Condition (D1) requires convexity with respect to market size up to $k$. Condition (D4) states that for bundles $T$ with at least $k$ firms the total amount $v(N)$ can be distributed among all firms in such a way that bundle $T$ receives at least the buyer's valuation for bundle $T$ and firms outside $T$ receive at least their marginal contribution to the grand bundle $N$. For bundles $T$ with $k-1$ firms this way of distributing the total amount $v(N)$ is not possible (by condition (D3)) unless the marginal contribution to the grand bundle $N$ of an arbitrary firm outside $T$ is replaced by its maximal marginal contribution with respect to those bundles, which can be obtained from $T$ by removing one good (condition (D2)).

As in the convex case, if $v$ is $k$-convex then it is monotonic with respect to market size up to $k-1$, i.e., $v(S) \leq v(T)$ for all $S \subseteq T \subseteq N$ with $|T| \leq k-1$.

According to the above definition, $v$ is 1-convex if and only if for all $S \subseteq N$ with $S \neq \emptyset, 0 \leq g(N) \leq g(S)$, which coincides with property PS but for the case $S=\emptyset$. Moreover, if $k=0$, then the above definitions amount to property PS.

Proposition 9 If the value function is monotonic and $k$-convex, then $(\widetilde{S}, \widetilde{\pi}) \in$ $S P E$-outcome set if and only if $\widetilde{S} \in \operatorname{sol}(\mathbf{L P})$ and $\left(0, \widetilde{\pi}^{S}\right) \in \operatorname{sol}(\mathbf{R D L P})$. 
Proof: See Appendix.

As above we characterize next the set of equilibrium price vectors as the convex hull of at most $n$ ! corner solutions of RDLP. When $v$ is monotonic and $k$-convex, then $n-k$ firms set prices equal to their marginal contributions and the other firms set prices below them. Thus, in the "0-convex" case (concavity) all the $S P E$-price vectors are the marginal contribution vector; in the 1-convex case $n-1$ firms set prices at their marginal contributions; and so on.

Definition 9 Let $v$ be a value function, $\sigma \in \Sigma$ and $k \in N$. The marginal contribution vector $x^{\sigma, k}(v) \in R^{n}$ of value function $v$ with respect to number $k$ and ordering $\sigma$ is given by,

$$
x_{i}^{\sigma, k}(v)= \begin{cases}v\left(P_{i}^{\sigma}+i\right)-v\left(P_{i}^{\sigma}\right) & \text { if } \sigma(i)<k \\ c^{*}(i)+g\left(P_{i}^{\sigma}\right)-g(N) & \text { if } \sigma(i)=k \\ c^{*}(i) & \text { if } \sigma(i)>k\end{cases}
$$

Proposition 10 Let $v$ be a monotonic, $k$-convex value function. The following three sets are the same.

(i) $\operatorname{Core}(v)$

(ii) SPE-price vector set

(iii) $\operatorname{conv}\left\{x^{\sigma, k}(v) \mid \sigma \in \Sigma\right\}$

Proof: (i) $\Longleftrightarrow$ (ii) is given by Propositions 4 and 9 jointly with the fact that if the buyer surplus is zero, then the set of solutions of RDLP is $\operatorname{core}(v) \subset \operatorname{core}(G)$.

$(\mathrm{i}) \Longleftrightarrow$ (iii) is show in Driessen (1993).

A $k$-convex value function $v$, with $k \leq n-1$, is symmetric ${ }^{5}$ if $v(S)=v(T)$ for all $S, T \subset N$, with $k \leq|S|=|T| \leq n-2$. When the value function $v$ is $k$-convex but $k<n$, it cannot be guaranteed that the Shapley value is in Core $(v)$ unless $v$ is symmetric for those bundles of size bigger than or equal to $k$, and satisfies that the highest marginal contribution is that of the grand coalition, i.e., $\frac{\sum_{S} c^{*}(i)}{|S|} \leq \frac{\sum_{N} c^{*}(i)}{|N|}$, for all $S \subseteq N$, denoted as property $(C S)$.

\footnotetext{
${ }^{5} \mathrm{~A}$ single product Bertrand competition is a particular case where the value function is symmetric but not $k$-convex. To see this, suppose that $v(S)=1$ if $S \neq \emptyset$ and $v(\emptyset)=0$, i.e., all firms produce the same product.
} 
Corollary 7 Let $v$ be a symmetric $k$-convex value function satisfying $C S$. Then the Shapley value of $v, \phi^{k}(v)$, which belongs to the core of $v$, is

$$
\phi_{i}^{k}(v)=\frac{1}{n !}\left[\sum_{\sigma \in \Sigma} x_{i}^{\sigma, k}(v)+(n-2) !(n-k-1)\left(c^{*}(N)-n c^{*}(i)\right] \quad \forall i \in N\right.
$$

and it is an SPE-price vector.

Proof: By Proposition 10, and Driessen (1993).

In addition, suppose that a price vector belonging to the core is suggested to firms by a central planner and that some subsets of firms may complain about their suggested prices. The nucleolus consists of all price vectors that minimize the maximal complaints of all subsets of firms (if all complaints could be arranged in the lexicographic order). The nucleolus is in the core of $v$ if it is not empty. In fact, for $k$-convex value functions the nucleolus exists and it is unique although it does not have an easy general expression unless $k=1$. Thus, we show that, since $v(N) \leq \sum_{N} c^{*}(k)$ in this case, firms set prices at their marginal contribution reduced by a constant: the gap of $N$ divided by $n$. This is the centre of gravity of the extreme points of core of $v$ and coincides with its nucleolus when $k=1$.

Corollary 8 Let $v$ be a monotonic, 1-convex value functions. Then firm i's component of the nucleolus of $v$ is given by

$$
c^{*}(i)-\frac{1}{n} g(N)=c^{*}(i)-\frac{1}{n}\left[\sum_{N} c^{*}(k)-v(N)\right]
$$

and it is an SPE-price of firm $i$.

Proof: By Proposition 2, the set of $S P E$-price vectors is non-empty, thus, by Proposition 10, it coincides with core $(v)$. Also, it is well known that if $\operatorname{core}(v)$ is non empty, then the nucleolus belongs to it. Thus, the nucleolus is a $S P E$-price vector.

ExAmple 5: Let $N=\{1,2,3\}$, and let the value function $v$ be,

\begin{tabular}{c|ccccccc}
$S$ & 1 & 2 & 3 & 1,2 & 1,3 & 2,3 & $1,2,3$ \\
\hline$v$ & 6 & 5 & 5 & $\alpha$ & $\alpha$ & $\beta$ & $\gamma$
\end{tabular}


Case 1: $v$ is 1-convex.

If $\alpha=30, \beta=20$ and $\gamma=42$, then it can be proven that $v$ is 1 -convex. Next table shows the vectors $x^{\sigma, 1}(v)$ for all $\sigma \in \Sigma$.

\begin{tabular}{cccc}
\hline$\sigma$ & $x_{1}^{\sigma, 1}(v)$ & $x_{2}^{\sigma, 1}(v)$ & $x_{3}^{\sigma, 1}(v)$ \\
\hline$\{1,2,3\}$ & 18 & 12 & 12 \\
$\{1,3,2\}$ & 18 & 12 & 12 \\
$\{2,1,3\}$ & 22 & 8 & 12 \\
$\{2,3,1\}$ & 22 & 8 & 12 \\
$\{3,1,2\}$ & 22 & 12 & 8 \\
$\{3,2,1\}$ & 22 & 12 & 8 \\
\hline
\end{tabular}

All of them are $S P E$-price vectors and by Proposition 10, their convex hull is the $S P E$-price vector set. The nucleolus is their average: $(22,12,12)-$ $\frac{1}{3}(4,4,4)=\left(\frac{62}{3}, \frac{32}{3}, \frac{32}{3}\right)$. Notice that $p^{b}=0$.

Case 2: $v$ is convex.

If $\alpha=30, \beta=20$ and $\gamma=60$, then $v$ is convex. Next table shows the vectors $x^{\sigma}(v)$ for all $\sigma \in \Sigma$.

\begin{tabular}{cccc}
\hline$\sigma$ & $x_{1}^{\sigma}(v)$ & $x_{2}^{\sigma}(v)$ & $x_{3}^{\sigma}(v)$ \\
\hline$\{1,2,3\}$ & 6 & 24 & 30 \\
$\{1,3,2\}$ & 6 & 30 & 24 \\
$\{2,1,3\}$ & 25 & 5 & 30 \\
$\{2,3,1\}$ & 40 & 5 & 15 \\
$\{3,1,2\}$ & 25 & 30 & 5 \\
$\{3,2,1\}$ & 40 & 15 & 5 \\
\hline
\end{tabular}

Again, all of them are $S P E$-price vectors and by Proposition 10 their convex hull is the $S P E$-price vector set. The nucleolus coincides with the Shapley value $\phi=\left(\frac{142}{6}, \frac{109}{6}, \frac{109}{6}\right)$.

Case 3: $v$ is concave.

If $\alpha=6, \beta=5$ and $\gamma=6$, then $v$ is concave, thus, the unique $S P E$ price vector is the marginal contributions, $(1,0,0)$ and the buyer's surplus is $p^{b}=5$. 


\section{The nonzero cost case}

Assume that the buyer's value function $v$, can take on positive as well as negative values. We have assumed up to now that firm $i$ 's (constant) cost of production, $c_{i}$, is zero. Let us consider a model in which these costs are positive, $c=\left(c_{1}, \ldots, c_{n}\right) \in R_{+}^{n}$. In this model with costs $(S, p)$ is an SPEoutcome if conditions C1, C2 of Proposition 1 are satisfied and condition C3 is modified as follows,

(C3') for every $A \subseteq N \backslash S$ and for all $T \supseteq A$

$$
v(S)-\sum_{k \in S} p_{k} \geq v(T)-\sum_{k \in T \backslash A} p_{k}-\sum_{k \in A} c_{k}
$$

Let $(v-c)(S)=v(S)-\sum_{k \in S} c_{k}$. The associated linear programming problem is the same as for the zero-cost case. It suffices to substitute the objective value function $v$ by $\{(v-c)\}$, both in the primal problem and on the right-hand side constraints of the corresponding dual problem. As in TUW(1997), all the results of the zero-cost case carry on to the non-zerocost case. The next Proposition relates equilibrium outcomes in the zero-cost model to those in the nonzero cost model. We reproduce some of them for completeness.

Proposition $11(S, p)$ is an SPE-outcome of the zero-cost model with value function $v$ if and only if $(S, p+c)$ is an $S P E$-outcome of the nonzero cost model in which the value function is $v+c$.

This Proposition asserts that the $S P E$-outcomes for the zero-cost case perfectly identify the equilibrium outcomes in the general model. If we are interested in the equilibrium outcomes of a model where the value function is $v$ and the cost vector $c$, we must find first the equilibria of a model with value function $v-c$ and costs of production equal to zero. If $(S, p)$ is an equilibrium outcome for the latter model then $(S, p+c)$ is an equilibrium outcome for the former model.

Proof: It suffices to prove that $(S, p)$ satisfies conditions $\mathrm{C} 1, \mathrm{C} 2$ and $\mathrm{C} 3$ of Proposition 1 in the model with value function $v$ and zero-costs if and only if $(S, p+c)$ satisfies conditions $\mathrm{C} 1, \mathrm{C} 2$ and $\mathrm{C} 3$ ', respectively, in the model with 
value function $v+c$ and $\operatorname{costs} c$. We will prove only the equivalence between C3 and C3' given that the other conditions' equivalence are similarly verified.

Let $A \subseteq N \backslash S$ and $T \supseteq A$,

$$
\begin{gathered}
v(S)-\sum_{k \in S} p_{k} \geq v(T)-\sum_{k \in T \backslash A} p_{k} \Longleftrightarrow \\
v(S)+\sum_{k \in S} c_{k}-\sum_{k \in S} p_{k}-\sum_{k \in S} c_{k} \geq v(T)+\sum_{k \in T} c_{k}-\sum_{k \in T \backslash A} p_{k}-\sum_{k \in T} c_{k} \Longleftrightarrow \\
(v+c)(S)-\sum_{k \in S}\left(p_{k}-c_{k}\right) \geq(v+c)(T)-\sum_{k \in T \backslash A}\left(p_{k}+c_{k}\right)-\sum_{k \in A} c_{k} .
\end{gathered}
$$

By Proposition 11 all the results for the zero-cost model can be generalized to the cost model. We offer an existence result,

Corollary 9 For every value function $v$ and cost vector $c$ there exists an SPE-outcome. Furthermore, $\widetilde{S}$ is an SPE-consumption set if and only if $\widetilde{S} \in \arg \max _{S \subseteq N}(v-c)(S)$.

Proof: Let $(\widetilde{S}, \widetilde{p})$ be an $S P E$-outcome for the zero-cost model where the value function is $v-c$. Then, by Proposition $11(\widetilde{S}, \widetilde{p}+c)$ is an $S P E$-outcome for the model with value function $v$ and production $\operatorname{costs} c$.

Moreover, by Lemma 4 every $S P E$-consumption set of the zero-cost model and value function $v-c$ is such that maximizes $(v-c)(S)$ over $S \subseteq N$. Hence, by Proposition 11 this is also the property of every equilibrium consumption set of the general model.

\section{Appendix}

\section{Proof of Proposition 2:}

Step 1: If $\left(\widetilde{\pi}^{b}, \tilde{\pi}\right) \in \operatorname{sol}(\mu-\mathrm{DLP})$ for some $\mu \in \Gamma$, then $\widetilde{\pi} \geq 0$ and $\widetilde{\pi}_{j}=0$, for all $j \in N \backslash \widetilde{S}$. Thus, $\widetilde{\pi}^{b}+\sum_{i \in N} \widetilde{\pi}_{i}=\widetilde{\pi}^{b}+\sum_{i \in \widetilde{S}} \widetilde{\pi}_{i}=v(\widetilde{S})$, which implies that, $v(\widetilde{S})-\sum_{i \in \widetilde{S}} \widetilde{\pi}_{i}=\widetilde{\pi}^{b}$. By the constraints of $\mu$-DLP, $\widetilde{\pi}^{b} \geq v(S)-\sum_{i \in S} \widetilde{\pi}_{i}$, for all $S \subseteq N$. Thus, 


$$
v(\widetilde{S})-\sum_{i \in \widetilde{S}} \widetilde{\pi}_{i}=\widetilde{\pi}^{b} \geq v(S)-\sum_{i \in S} \widetilde{\pi}_{i} \quad S \subseteq N
$$

and $\mathrm{C} 1$ is satisfied.

Step 2: To prove that C2 is satisfied consider the following two cases:

Case 1: $\widetilde{\pi}^{b}=0$. Given that $v(\widetilde{S})-\sum_{i \in \widetilde{S}} \widetilde{\pi}_{i}=\widetilde{\pi}^{b}=0$, then $\mathrm{C} 2$ holds for $S_{i}=\emptyset$ and for all $i \in \widetilde{S}$.

Case 2: $\widetilde{\pi}^{b}>0$. Suppose there exists $j \in \widetilde{S}$ such that for all $S_{j} \subseteq N \backslash\{j\}$ it is verified that,

$$
\widetilde{\pi}^{b}=v(\widetilde{S})-\sum_{i \in \widetilde{S}} \widetilde{\pi}_{i}>v\left(S_{j}\right)-\sum_{i \in S_{j}} \widetilde{\pi}_{i}
$$

We will see that this implies that there exists an $\epsilon>0$ such that $\left(\widetilde{\pi}^{b}-\right.$ $\left.\epsilon, \widetilde{\pi}_{1}, \ldots, \widetilde{\pi}_{j-1}, \widetilde{\pi}_{j}+\epsilon, \widetilde{\pi}_{j+1}, \ldots, \widetilde{\pi}_{n}\right)$ is a solution of the $\mu$-DLP, which contradicts the optimality of $\sum_{l=1}^{L}\left(\sum_{i \in S_{l}} \tilde{\pi}_{i}\right) 10^{d(L-l)}$ and then there cannot exist any $j \in \widetilde{S}$ as described above.

Let $\alpha=\max _{S \subseteq N \backslash\{j\}}\left(v(S)-\sum_{i \in S} \tilde{\pi}_{i}\right)$, take $\epsilon=\left(\widetilde{\pi}^{b}-\alpha\right) / 2$. Let us see that $\left(\widetilde{\pi}^{b}-\epsilon, \widetilde{\pi}_{1}, \ldots, \widetilde{\pi}_{j-1}, \widetilde{\pi}_{j}+\epsilon, \widetilde{\pi}_{j+1}, \ldots, \widetilde{\pi}_{n}\right)$ verifies all the constraints of the restricted dual problem.

Let $S \subseteq N$, if $j \in S$, then $\left(\widetilde{\pi}^{b}-\epsilon\right)+\sum_{i \in S \backslash\{j\}} \tilde{\pi}_{i}+\left(\widetilde{\pi}_{j}+\epsilon\right)=\widetilde{\pi}^{b}+\sum_{i \in S} \widetilde{\pi}_{i} \geq$ $v(S)$. If $j \notin S$ given that $v(S)-\sum_{i \in S} \widetilde{\pi}_{i} \leq \alpha=\widetilde{\pi}^{b}-2 \epsilon \leq \widetilde{\pi}^{b}-\epsilon$, then $\left(\widetilde{\pi}^{b}-\epsilon\right)+\sum_{i \in S} \widetilde{\pi}_{i} \geq v(S)$.

With respect to the last constraint,

$$
v(\widetilde{S})=\widetilde{\pi}^{b}+\sum_{i \in \widetilde{S}} \widetilde{\pi}_{i}=\left(\widetilde{\pi}^{b}-\epsilon\right)+\sum_{i \in \widetilde{S} \backslash\{j\}} \tilde{\pi}_{i}+\left(\widetilde{\pi}_{j}+\epsilon\right)
$$

Hence, $\left(\widetilde{\pi}^{b}-\epsilon, \widetilde{\pi}_{1}, \ldots, \widetilde{\pi}_{j-1}, \widetilde{\pi}_{j}+\epsilon, \widetilde{\pi}_{j+1}, \ldots, \widetilde{\pi}_{n}\right)$ verifies all the constraints of $\mu$-DLP. Moreover, suppose that $\mu=\left\{S_{1}, \ldots, S_{L}\right\}$ and that $j \in S_{l^{\prime}}$. Then,

$$
\begin{aligned}
F\left(\mu,\left(\widetilde{\pi}_{1}, \ldots, \widetilde{\pi}_{j-1}, \widetilde{\pi}_{j}+\epsilon, \widetilde{\pi}_{j+1}, \ldots, \widetilde{\pi}_{N}\right)\right) & =\sum_{l=1}^{L}\left(\sum_{i \in S_{l}} \widetilde{\pi}_{i}\right) 10^{d(L-l)}+\epsilon 10^{d\left(L-l^{\prime}\right)} \\
& >\sum_{l=1}^{L}\left(\sum_{i \in S_{l}} \widetilde{\pi}_{i}\right) 10^{d(L-l)} \\
& =F\left(\mu,\left(\widetilde{\pi}_{1}, \ldots, \widetilde{\pi}_{j-1}, \widetilde{\pi}_{j}, \widetilde{\pi}_{j+1}, \ldots, \widetilde{\pi}_{N}\right)\right)
\end{aligned}
$$


which contradict that $\left(\widetilde{\pi}^{b}, \tilde{\pi}\right) \in \operatorname{sol}(\mu$-DLP $)$.

Step 3: To see C3, let $A \subseteq N \backslash \widetilde{S}$ and let $T \supseteq A$. Given that $\widetilde{\pi}_{i}=0$ if $i \in A$ (Lemma 3), then

$$
\begin{aligned}
v(\widetilde{S})-\sum_{i \in \widetilde{S}} \tilde{\pi}_{i} \geq v(T)-\sum_{i \in T} \widetilde{\pi}_{i}=v(T)-\sum_{i \in T \cap A} \tilde{\pi}_{i}-\sum_{i \in T \backslash A} \tilde{\pi}_{i} \\
=v(T)-\sum_{i \in T \backslash A} \tilde{\pi}_{i} .
\end{aligned}
$$

Proof of Proposition 3: The proof consists of several steps.

Step 1: Given that $(0, \widetilde{\pi})$ is a solution of DLP then $\widetilde{\pi} \geq 0$ and $\forall j \in N \backslash \widetilde{S}$, and by Lemma $3, \widetilde{\pi}_{j}=0$. Thus, $\sum_{i \in N} \widetilde{\pi}_{i}=\sum_{i \in \widetilde{S}} \widetilde{\pi}_{i}=v(\widetilde{S})$, which implies that $v(\widetilde{S})-\sum_{i \in \widetilde{S}} \widetilde{\pi}_{i}=0$ and hence $(0, \widetilde{\pi}) \in \operatorname{sol}(\mathbf{R D L P})$.

Now, let us consider $(\widetilde{S}, \pi)$ where $\pi_{i}=\widetilde{\pi}_{i}$ for all $i \in \widetilde{S}$ and $\pi_{N \backslash \widetilde{S}} \in R_{+}^{|N \backslash \widetilde{S}|}$. We have to show that $(\widetilde{S}, \pi) \in S P E$-outcome set.

On the one hand, $v(\widetilde{S})-\sum_{i \in \widetilde{S}} \pi_{i}=v(\widetilde{S})-\sum_{i \in \widetilde{S}} \widetilde{\pi}_{i}=0$. On the other, for all $S \subseteq N$,

$$
\sum_{i \in S} \pi_{i} \geq \sum_{i \in S} \tilde{\pi}_{i} \geq v(S)
$$

where the first inequality holds by the definition of $\pi_{i}$ and the second by the constraints of RDLP. Thus,

$$
v(\widetilde{S})-\sum_{i \in \widetilde{S}} \pi_{i}=0 \geq v(S)-\sum_{i \in S} \tilde{\pi}_{i} \geq v(S)-\sum_{i \in S} \pi_{i} \quad S \subseteq N
$$

and $\mathrm{C} 1$ is verified.

Step 2: Given that $v(\widetilde{S})-\sum_{i \in \widetilde{S}} \pi_{i}=0, \mathrm{C} 2$ is verified for $S_{i}=\emptyset$, for all $i \in \widetilde{S}$.

Step 3: Finally, to prove C3, let $A \subseteq N \backslash \widetilde{S}$ a let $T \supseteq A$. Given that $\widetilde{\pi}_{i}=0$ if $i \in A$ (Lemma 3), then

$$
\begin{aligned}
v(\widetilde{S})-\sum_{i \in \widetilde{S}} \pi_{i} & \geq v(T)-\sum_{i \in T} \tilde{\pi}_{i}=v(T)-\sum_{i \in T \cap A} \tilde{\pi}_{i}-\sum_{i \in T \backslash A} \tilde{\pi}_{i}= \\
& =v(T)-\sum_{i \in T \backslash A} \widetilde{\pi}_{i} \geq v(T)-\sum_{i \in T \backslash A} \pi_{i} .
\end{aligned}
$$


To prove proposition 4 we need first the following result:

Proposition 12 Let $v$ be a value function. $\left(\widetilde{S}, \widetilde{p}^{\widetilde{S}}\right) \in S P E$-outcome set if and only if

i) $\widetilde{S} \in \operatorname{sol}(\mathbf{L P})$,

ii) $\left(\widetilde{\pi}^{b}, \widetilde{\pi}^{\widetilde{S}}\right) \in \operatorname{sol}(\mathbf{D L P})$, where $\widetilde{\pi}^{b}=v(\widetilde{S})-\sum_{k \in \widetilde{S}} \widetilde{p}_{k}$, and $\widetilde{\pi}^{\widetilde{S}}=\widetilde{p}^{\widetilde{S}}$.

iii) It does not exits any other $\left(\pi^{b}, \pi^{\widetilde{S}}\right) \in \operatorname{sol}(\mathbf{D L P})$, such that $\pi^{\widetilde{S}}$ weakly Pareto-dominates $\widetilde{\pi}^{\widetilde{S}}$.

Proof: Let $\left(\widetilde{S}, \widetilde{p}^{\widetilde{S}}\right)$ be in the set of $S P E$-outcomes. By Lemma $4, \widetilde{S} \in$ $\operatorname{sol}(\mathbf{L P})$. Also, it can be easily checked that $S P E$-price vectors satisfying (C1)-(C3) verify all the constraints of the linear programming problem. Thus, any $S P E$-price vector where non-active firms set prices equal to marginal costs is a solution of DLP, and then $\left(\widetilde{\pi}^{b}, \widetilde{\pi}^{S}\right) \in \operatorname{sol}(\mathbf{D L P})$ where $\widetilde{\pi}^{b}=v(\widetilde{S})-$ $\sum_{\widetilde{S}} \widetilde{p}_{k}$, and $\widetilde{\pi}^{\widetilde{S}}=\widetilde{p}^{\widetilde{S}}$. Moreover, by Lemma $6, \widetilde{\pi}^{\widetilde{S}}$ is non-Pareto dominated by any other $S P E$-price vector.

Now, suppose that the three conditions in the Proposition are satisfied.

Step 1: By condition (ii) $\left(\widetilde{\pi}^{b}, \widetilde{\pi}^{S}\right) \in \operatorname{sol}(\mathbf{D L P})$, then $\widetilde{\pi}^{\widetilde{S}} \geq 0$ and $\widetilde{\pi}_{j}=0$ for all $j \in N \backslash \widetilde{S}$. Thus, $\widetilde{\pi}^{b}+\sum_{i \in N} \widetilde{\pi}_{i}^{\widetilde{S}}=\widetilde{\pi}^{b}+\sum_{i \in \widetilde{S}} \widetilde{\pi}_{i}^{\widetilde{S}}=v(\widetilde{S})$, which implies that, $\widetilde{\pi}^{b}=v(\widetilde{S})-\sum_{i \in \widetilde{S}} \widetilde{\pi}_{i}$.

By the constraints of DLP,

$$
\widetilde{\pi}^{b} \geq v(S)-\sum_{i \in S} \widetilde{\pi}_{i}^{\widetilde{S}}, \text { for all } S \subseteq N
$$

Thus, for all $S \subseteq N$,

$$
v(\widetilde{S})-\sum_{i \in \widetilde{S}} \widetilde{\pi}_{i}^{\widetilde{S}} \geq v(S)-\sum_{i \in S} \widetilde{\pi}_{i}^{\widetilde{S}}
$$

and $\mathrm{C} 1$ is verified.

Step 2: To prove that $\mathrm{C} 2$ is satisfied we consider two cases:

Case 1: $\widetilde{\pi}^{b}=0$. Given that $v(\widetilde{S})-\sum_{i \in \widetilde{S}} \widetilde{\pi}_{i}^{\widetilde{S}}=\widetilde{\pi}^{b}=0$ then C2 holds for $S_{i}=\emptyset$ and for all $i \in \widetilde{S}$. 
Case 2: $\widetilde{\pi}^{b}>0$. Suppose there exists $j \in \widetilde{S}$ such that for all $S_{j} \subseteq N \backslash\{j\}$,

$$
\widetilde{\pi}^{b}=v(\widetilde{S})-\sum_{i \in \widetilde{S}} \widetilde{\pi}_{i}^{\widetilde{S}}>v\left(S_{j}\right)-\sum_{i \in S_{j}} \widetilde{\pi}_{i}^{\widetilde{S}}
$$

is verified. We show that this implies that there exists an $\epsilon>0$ such that $\left(\widetilde{\pi}^{b}-\epsilon, \widetilde{\pi}_{1}^{\widetilde{S}}, \ldots, \widetilde{\pi}_{j-1}^{\widetilde{S}}, \widetilde{\pi}_{j}^{\widetilde{S}}+\epsilon, \widetilde{\pi}_{j+1}^{S}, \ldots, \widetilde{\pi}_{n}^{S}\right)$ is a solution of the DLP, which contradicts assumption iii):

Let $\alpha=\max _{S \subseteq N \backslash\{j\}}\left\{v(S)-\sum_{i \in S} \widetilde{\pi}_{i}^{\widetilde{S}}\right\}$, take $\epsilon=\left(\widetilde{\pi}^{b}-\alpha\right) / 2$. Let us see that $\left(\widetilde{\pi}^{b}-\epsilon, \widetilde{\pi}_{1}^{\widetilde{S}}, \ldots, \widetilde{\pi}_{j-1}, \widetilde{\pi}_{j}+\epsilon, \widetilde{\pi}_{j+1}, \ldots, \widetilde{\pi}_{n}^{\widetilde{S}}\right)$ verifies all the constraints of the restricted dual problem.

Let $S \subseteq N$, if $j \in S$, then

$$
\left(\widetilde{\pi}^{b}-\epsilon\right)+\sum_{i \in S \backslash\{j\}} \widetilde{\pi}_{i}^{\widetilde{S}}+\left(\widetilde{\pi}_{j}^{\widetilde{S}}+\epsilon\right)=\widetilde{\pi}^{b}+\sum_{i \in S} \widetilde{\pi}_{i}^{\widetilde{S}} \geq v(S) .
$$

If $j \notin S$, given that $v(S)-\sum_{i \in S} \widetilde{\pi}_{i}^{\widetilde{S}} \leq \alpha=\widetilde{\pi}^{b}-2 \epsilon \leq \widetilde{\pi}^{b}-\epsilon$, we have

$$
\left(\widetilde{\pi}^{b}-\epsilon\right)+\sum_{i \in S} \widetilde{\pi}_{i}^{\widetilde{S}} \geq v(S) .
$$

Finally, the objective value function is,

$$
\left(\widetilde{\pi}^{b}-\epsilon\right)+\sum_{i \in \widetilde{S} \backslash\{j\}} \widetilde{\pi}_{i}^{\widetilde{S}}+\left(\widetilde{\pi}_{j}^{\widetilde{S}}+\epsilon\right)=\widetilde{\pi}^{b}+\sum_{i \in \widetilde{S}} \widetilde{\pi}_{i}^{\widetilde{S}}=v(\widetilde{S}) .
$$

Hence, $\left(\widetilde{\pi}^{b}-\epsilon, \widetilde{\pi}_{1}^{\widetilde{S}}, \ldots, \widetilde{\pi}_{j-1}^{\widetilde{S}}, \widetilde{\pi}_{j}^{\widetilde{S}}+\epsilon, \widetilde{\pi}_{j+1}^{\widetilde{S}}, \ldots, \widetilde{\pi}_{n}^{\widetilde{S}}\right) \in \operatorname{sol}(\mathbf{D L P})$ which contradicts assumption iii).

Step 3: Given that $\widetilde{\pi}_{j}^{\widetilde{S}}=0$ for all $j \in N \backslash \widetilde{S}$, then trivially C3 is satisfied. Hence, $(\widetilde{S}, \widetilde{p} \widetilde{S}) \in S P E$-outcome set, where $\widetilde{\pi}^{\widetilde{S}}=\widetilde{p}^{\widetilde{S}}$.

Proof of Proposition 4: The set of optimal solutions of DLP is a convex polyhedron, and so is the subset of not weakly Pareto-dominated price vectors. A polyhedral set is completely characterized by its vertices Hence, by the previous result it suffices to show that the set of non Pareto-dominated vectors $\widetilde{\pi}^{\widetilde{S}}$, for which there exists $\widetilde{\pi}^{b}$ such that $\left(\widetilde{\pi}^{b}, \widetilde{\pi}^{S}\right) \in \operatorname{sol}(\mathbf{D L P})$, coincide with the solutions of some $\mu$-DLP. 
Let $\left(\widetilde{\pi}^{b}, \widetilde{\pi}^{\widetilde{S}}\right) \in \operatorname{sol}(\mu-\mathrm{DLP})$. Trivially $\left(\widetilde{\pi}^{b}, \widetilde{\pi}^{\widetilde{S}}\right) \in \operatorname{sol}(\mathbf{D L P})$. Moreover, if there exists a $\left(\pi^{b}, \pi^{\tilde{S}}\right) \in \operatorname{sol}(\mathbf{D L P})$ such that $\pi^{\widetilde{S}}$ weakly Pareto-dominates $\widetilde{\pi}^{\widetilde{S}}$ then

$$
\begin{aligned}
F\left(\mu, \pi^{\tilde{S}}\right) & =\sum_{l=1}^{L}\left(\sum_{i \in S_{l}} \pi_{i}^{\widetilde{S}}\right) 10^{d(L-l)}> \\
& >\sum_{l=1}^{L}\left(\sum_{i \in S_{l}} \widetilde{\pi}_{i}^{\widetilde{S}}\right) 10^{d(L-l)}= \\
& =F\left(\mu, \widetilde{\pi}^{\widetilde{S}}\right)
\end{aligned}
$$

which contradicts that $\left(\widetilde{\pi}^{b}, \widetilde{\pi}^{\tilde{S}}\right) \in \operatorname{sol}\left(\mu\right.$-DLP). Thus if $\left(\widetilde{\pi}^{b}, \widetilde{\pi}^{\tilde{S}}\right) \in \operatorname{sol}(\mu$ DLP), then $\left(\widetilde{\pi}^{b}, \widetilde{\pi}^{\tilde{S}}\right) \in \operatorname{sol}(\mathbf{D L P})$ and it is not Pareto-dominated.

Now, let $\left(\widetilde{\pi}^{b}, \widetilde{\pi}^{\tilde{S}}\right) \in \operatorname{sol}(\mathbf{D L P})$, where $\widetilde{\pi}^{\tilde{S}}$ is not Pareto-dominated by the price vector of any other solution and where $\left(\widetilde{\pi}^{b}, \widetilde{\pi}^{\tilde{S}}\right)$ is a vertex solution (i.e., it is not the convex combination of other solutions). Then, there exists a set $S \subseteq N$ such that for any other $\left(\pi^{b}, \pi^{\widetilde{S}}\right) \in \operatorname{sol}(\mathbf{D L P})$ it is verified $\sum_{k \in S} \widetilde{\pi}_{k}^{\widetilde{S}}>\sum_{k \in S} \pi_{k}^{\widetilde{S}}$. Let $\mu \in \Gamma$ be an ordered partition such that $S_{1}=S$, clearly $\left(\widetilde{\pi}^{b}, \widetilde{\pi}^{\tilde{S}}\right) \in \operatorname{sol}(\mu$-DLP).

Hence, the weakly non-Pareto dominated convex hull of all the solutions of the $\mu$-DLP problems is the same set than that of solutions of DLP for which the price vector is weakly non-Pareto dominated.

For the proof of Proposition 6 it is shown first.

Lemma 12 If $(N, p) \in S P E$-outcome set, then $\sum_{S} p_{k} \leq c^{*}(S)$ for all $S \subseteq$ $N$

Proof: By C1, $v(N)-\sum_{k \in N} p_{k} \geq v(N \backslash S)-\sum_{k \in N \backslash S} p_{k}$, for all $S \subseteq N$, thus,

$$
\begin{aligned}
c^{*}(S) & =v(N)-v(N \backslash S) \geq \sum_{k \in N} p_{k}-\sum_{k \in N \backslash S} p_{k} \\
& =\sum_{k \in S} p_{k} .
\end{aligned}
$$


In particular, the above Lemma implies that $p_{k} \leq c^{*}(k)$ for all $k \in N$.

\section{Proof of Proposition 6:}

Step 1: $\left(N, p^{*}\right) \in S P E$-outcome set, where $p_{i}^{*}=c^{*}(i)$.

By monotonicity $p_{i}^{*} \geq 0$. By the 'products substitution' condition, $v(N)-$ $v(S) \geq \sum_{i \notin S} v(N)-v(N \backslash i)=\sum_{i \in N \backslash S} p_{i}^{*}$, for all $S \subseteq N$, or equivalently that $v(N)-\sum_{i \in N} p_{k}^{*} \geq v(S)-\sum_{i \in S} p_{k}^{*}$. Thus, C1 holds.

$\mathrm{C} 2$ is trivially satisfied for $S_{j}=N \backslash j$.

Step 2: If $(N, p) \in S P E$-outcome set, then $p_{i}=p_{i}^{*}$.

By Lemma $12, p_{i} \leq c^{*}(i)=p_{i}^{*}$. Suppose that there exists $j$ such that $p_{j}<c^{*}(j)$. By step 1 and $\mathrm{C} 1, v(N)-v(S) \geq \sum_{k \in N \backslash S} p_{k}^{*}>\sum_{k \in N \backslash S} p_{k}$, for all $S \subseteq N \backslash j$. Thus, $v(N)-\sum_{k \in N} p_{k}>v(S)-\sum_{k \in S} p_{k}$, for all $S \subseteq N \backslash j$. But then, firm $j$ has an incentive to raise its price, which is a contradiction. Hence $p_{j}=c^{*}(j)$.

Step 3: Let us prove that if $(S, p) \in S P E$-outcome set, then $\left(N, p^{S}\right) \in$ $S P E$-outcome set.

If $(S, p) \in S P E$-outcome set, then $\left(S, p^{S}\right) \in S P E$-outcome set. By C1,

$$
v(S)-\sum_{k \in S} p_{k}^{S} \geq v(N)-\sum_{k \in N} p_{k}^{S}=v(N)-\sum_{k \in S} p_{k}^{S}
$$

Given that $v$ is monotonic,

$$
v(S)-\sum_{k \in S} p_{k}^{S} \leq v(N)-\sum_{k \in S} p_{k}^{S}
$$

Therefore, by the two above expressions,

$$
v(S)-\sum_{k \in S} p_{k}^{S}=v(N)-\sum_{k \in N} p_{k}^{S} \geq v(T)-\sum_{k \in T} p_{k}^{S}
$$

for all $T \subseteq N$. Hence $\left(N, p^{S}\right)$ satisfies $\mathrm{C} 1$. To show that $\mathrm{C} 2$ also holds if $i \in N \backslash S$, let $S_{i}=S$, but if $i \in S$, then let $S_{i}$ the one given by the fact that $\left(S, p^{S}\right) \in S P E$-outcome set.

Step 4: Now, we are ready to prove Proposition 6. If conditions (i) and (ii) of the Proposition are verified then by Proposition $2,(\widetilde{S}, \widetilde{\pi}) \in S P E$-outcome set. 
Let us consider that $(\widetilde{S}, \widetilde{\pi}) \in S P E$-outcome set. By Lemma $4, \widetilde{S} \in$ $\arg \max _{S \subseteq N} v(S)$, hence $\widetilde{S} \in \operatorname{sol}(\mathbf{L P})$ and condition (i) is satisfied. Moreover, by monotonicity of $v, v(N) \geq v(\widetilde{S})$ which implies that $v(N)=v(\widetilde{S})$.

Let us show condition (ii): if $(\widetilde{S}, \widetilde{\pi}) \in S P E$-outcome set, then $\left(N, \widetilde{\pi}^{\widetilde{S}}\right) \in$ $S P E$-outcome set (step 3). But then it must be the case that $p^{*}=\widetilde{\pi}^{S}$ (step $2)$.

Let $\widetilde{\pi}^{b}=v(\widetilde{S})-\sum_{i \in \widetilde{S}_{i}} \widetilde{\pi}_{i}$, then $\left(\widetilde{\pi}^{b}, \widetilde{\pi}^{\widetilde{S}}\right)$ is a solution of DLP: first, $\widetilde{\pi}^{b} \geq 0$ given that $(\widetilde{S}, \widetilde{\pi}) \in S P E$-outcome set and by $\mathrm{C} 1, \widetilde{\pi}^{b} \geq v(S)-\sum_{k \in S} \widetilde{\pi}_{k}^{\widetilde{S}}$ for all $S \subseteq N$. To show that $\left(\widetilde{\pi}^{b}, \widetilde{\pi}^{\widetilde{S}}\right)$ is a solution of RDLP consider any $\left(\widehat{\pi}^{b}, \widehat{\pi}\right) \in \operatorname{sol}(\mathbf{R D L P})$. Then $(\widetilde{S}, \widehat{\pi}) \in S P E$-outcome set, thus by step 3 $\left(N, \widehat{\pi}^{\widetilde{S}}\right) \in S P E$-outcome set and by step $2, \widehat{\pi}^{\widetilde{S}}=p^{*}$. In addition, given that $\widehat{\pi}_{i}=0$, for all $i \in N \backslash \widetilde{S}$, we have that $\widehat{\pi}=p$. Thus, $\sum_{k \in N} \widehat{\pi}_{k}=\sum_{k \in N} p_{k}^{*}=$ $\sum_{k \in N} \widetilde{\pi}_{k}^{\widetilde{S}}$. Hence $\left(\widetilde{\pi}^{b}, \widetilde{\pi}^{\widetilde{S}}\right)=\left(\widehat{\pi}^{b}, \widehat{\pi}\right) \in \operatorname{sol}(\mathbf{R D L P})$.

Proof of Proposition 7: By Proposition 3, we know that if $\widetilde{S} \in \operatorname{sol}(\mathbf{L P})$ and $\left(0, \widetilde{\pi}^{S}\right) \in \operatorname{sol}(\mathbf{D L P})$, then $(\widetilde{S}, \widetilde{\pi}) \in S P E$-outcome set.

Let us prove that if $(\widetilde{S}, \widetilde{\pi}) \in S P E$-outcome set, then $\widetilde{S} \in \operatorname{sol}(\mathbf{L P})$ and $\left(0, \widetilde{\pi}^{S}\right) \in \operatorname{sol}(\mathbf{D L P})$. By Lemma $4, \widetilde{S} \in \arg \max _{S \subseteq N} v(S)$, which implies that $\widetilde{S} \in \operatorname{sol}(\mathbf{L P})$.

We prove that $\left(0, \widetilde{\pi}^{\widetilde{S}}\right)$ is a solution of $\mathbf{D L P}$ in several steps.

Step 1: First let us see that the set $S_{j}$ in C2 is the null set. Let $j \in \widetilde{S}$ and let $S_{j}$ be a minimal set, with respect to inclusion, verifying that $j \notin S_{j}$ and $v(\widetilde{S})-\sum_{\widetilde{S}} \widetilde{\pi}_{k}=v\left(S_{j}\right)-\sum_{S_{j}} \widetilde{\pi}_{k}$. If $S_{j} \neq \emptyset$, then let $k \in S_{j}$. If $k \in \widetilde{S}$, then let $S_{k}$ be the set that verifies $\mathrm{C} 2$ (thus $k \notin S_{k}$ ) and finally if $k \notin \widetilde{S}$, then let $S_{k}=\widetilde{S}$. Thus,

$$
\begin{aligned}
v(\widetilde{S})-\sum_{i \in \widetilde{S}} \widetilde{\pi}_{i} & \geq v\left(S_{j} \cup S_{k}\right)-\sum_{i \in S_{j} \cup S_{k}} \widetilde{\pi}_{i} \\
& \geq v\left(S_{j}\right)+v\left(S_{k}\right)-v\left(S_{j} \cap S_{k}\right)-\sum_{i \in S_{j}} \widetilde{\pi}_{i}-\sum_{i \in S_{k}} \widetilde{\pi}_{i}+\sum_{i \in S_{j} \cap S_{k}} \tilde{\pi}_{i} \\
& =2\left(v(\widetilde{S})-\sum_{i \in \widetilde{S}} \widetilde{\pi}_{i}\right)-\left(v\left(S_{j} \cap S_{k}\right)-\sum_{i \in S_{j} \cap S_{k}} \widetilde{\pi}_{i}\right)
\end{aligned}
$$


where the first inequality is given by the optimality of $\widetilde{S}$, the second by the convexity of $v$ and the last one by the properties of $S_{k}$ and $S_{j}$.

Hence, $v\left(S_{j} \cap S_{k}\right)-\sum_{i \in S_{j} \cap S_{k}} \widetilde{\pi}_{i} \geq v(\widetilde{S})-\sum_{i \in \widetilde{S}} \widetilde{\pi}_{i}$, and by optimality of $\widetilde{S}$ the above inequality translates into strict equality. However, this contradicts the minimality of $S_{j}\left(S_{j} \cap S_{k} \neq S_{j}\right.$ given that $k \in S_{j}$ and $k \notin S_{k}$; and if $S_{j} \cap S_{k}=\emptyset$, then the null set verifies C2 which contradicts again the minimality of $\left.S_{j}\right)$. Hence, $S_{j}=\emptyset$ and $v(\widetilde{S})=\sum_{i \in \widetilde{S}} \widetilde{\pi}_{i}=\sum_{i \in N} \widetilde{\pi}_{i}^{\widetilde{S}}$.

Step 2: Recall that if $(\widetilde{S}, \widetilde{\pi}) \in S P E$, then $\left(\widetilde{S}, \widetilde{\pi}^{S}\right) \in S P E$-outcome set. Now, let $S \subseteq N$, by C3 for $A \subseteq S \backslash \widetilde{S}$,

$$
\begin{aligned}
0 & =v(\widetilde{S})-\sum_{i \in \widetilde{S}} \widetilde{\pi}_{i}^{\widetilde{S}} \geq v(S)-\sum_{i \in S \backslash A} \widetilde{\pi}_{i}^{\widetilde{S}} \\
& =v(S)-\sum_{i \in S} \widetilde{\pi}_{i}^{\widetilde{S}}
\end{aligned}
$$

hence $\sum_{i \in S} \widetilde{\pi}_{i}^{\widetilde{S}} \geq v(S)$. Thus, $\left(0, \widetilde{\pi}^{\widetilde{S}}\right)$ is a solution of DLP. Moreover, by Step 1,

$$
\sum_{i \in N} \widetilde{\pi}_{i}^{\widetilde{S}}=\sum_{i \in S} \widetilde{\pi}_{i}^{\widetilde{S}}=v(\widetilde{S})
$$

hence $\left(0, \widetilde{\pi}^{\widetilde{S}}\right) \in \operatorname{sol}(\mathbf{R D L P}) \subseteq \operatorname{sol}(\mathbf{D L P})$.

Proof of Proposition 9: Let $v$ be a monotonic $k$-convex value function. By monotonicity, if $\widetilde{S} \in \operatorname{sol}(\mathbf{L P})$, then $v(\widetilde{S})=v(N)$. Now, by Proposition 3, if $\widetilde{S} \in \operatorname{sol}(\mathbf{L P})$ and $(0, \widetilde{\pi}) \in \operatorname{sol}(\mathbf{D L P})$, then $(\widetilde{S}, \widetilde{\pi}) \in S P E$-outcome set.

Let us prove that if $(\widetilde{S}, \widetilde{\pi}) \in S P E$-outcome set, then $\widetilde{S} \in \operatorname{sol}(\mathbf{L P})$ and $(0, \widetilde{\pi}) \in \operatorname{sol}(\mathbf{D L P})$. By Lemma $4, \widetilde{S} \in \arg \max _{S \subseteq N} v(S)$ which implies that $\widetilde{S} \in \operatorname{sol}(\mathbf{L P})$.

We prove that $(0, \tilde{\pi}) \in \operatorname{sol}(\mathbf{D L P})$ or equivalently that $(0, \tilde{\pi}) \in \operatorname{sol}(\mathbf{R D L P})$ : define

$$
v_{k}=\left\{\begin{array}{ccc}
v(S) & \text { if } & |S|<k \\
v(S)+g(S)-g(N) & \text { if } & |S| \geq k
\end{array}\right.
$$

Notice that $v_{k}(S) \geq v(S)$, for all $S \subseteq N$. Trivially $v_{k}(S) \geq v(S)$, for all $S \subseteq N$ with $|S|<k$. By (D1), $g(S)-g(N) \geq 0$, for all $S \subseteq N$ with $|S| \geq k$. Thus, $v_{k}(S)=v(S)+g(S)-g(N) \geq v(S)$.

The above implies that $v_{k}$ is the $k$-cover of $v$, it is convex and the core of the economy induced by $v$ and $v_{k}$ are the same (Driessen 1986). However, 
the core of the economy induced by $v_{k}$ is, by Proposition 8, the $S P E$-price vector set. Hence, let $(0, \widehat{\pi})$ be a solution of RDLP under value function $v_{k}$. Then, it is also a solution of RDLP under value function $v$.

Now, if $(\widetilde{S}, \widetilde{\pi}) \in S P E$-outcome set and $v$ is monotonic, then $(N, \widetilde{\pi}) \in$ $S P E$-outcome set. Thus, by Proposition $4,\left(\widetilde{\pi}^{b}, \widetilde{\pi}\right) \in \operatorname{sol}(\mathbf{D L P})$, where $\widetilde{\pi}^{b}=$ $v(N)-\sum_{N} \widetilde{\pi}_{i}$. If $\widetilde{\pi}^{b}>0$, then for all $\sigma \in \Sigma$, and $x^{\sigma, k}$ there exists a firm $i_{0}$ such that $x_{i_{0}}^{\sigma, k}=c^{*}(i)=v(N)-v(N \backslash i)<\tilde{\pi}_{i}$, which is a contradiction.

\section{References}

[1] Bikhchandani, S. and J.W. Mamer, 1997, Competitive Equilibrium in a Exchange Economy with Indivisibilities, Journal of Economic Theory, $74,385-413$.

[2] Bikhchandani, S. and J.M. Ostroy, 2001, The Package Assignment Model, Working Paper.

[3] d'Aspremont, C., Gabszewicz, J. and J.F. Thisse, 1979, On Hotelling's stability in competition, Econometrica, 17, 1145-1151.

[4] Dantzig, G.B., 1974, Linear Programming and Extensions, Princeton Univ. Press, Princeton, NJ.

[5] Driessen, T., 1986, Solution concepts of $k$-convex $n$-person games, International Journal of Game Theory, 15, 201-229.

[6] Driessen, T., 1988, Cooperative games, solutions and applications, Kluwer Academic Publishers, Dordrecht, The Netherlands.

[7] Driessen, T., 1993, Generalized concavity in game theory: characterizations in terms of the core, Memorandum No 1121, Faculty of Applied Mathematics. University of Twente.

[8] Driessen, T. and C. Rafels, C., 1999, Characterizations of $k$-convex games, Optimization, 46, 403-431.

[9] Gabszewicz, J. and J.F. Thisse, 1979, Price competition, quality and income disparities, Journal of Economic Theory, 20, 340-359. 
[10] Gabszewicz, J. and J.F. Thisse, 1980, Entry (and exit) in a differentiated industry, Journal of Economic Theory, 22, 327-338.

[11] Gul, F. and E. Stacchetti, 2000, The English Auction with Differentiated Commodities, Journal of Economic Theory, 92, 66-95.

[12] Hotelling, H., 1929, Stability in Competition, Economic Journal, 39, 41-57.

[13] Kelso, A. S. and V.P. Crawford, 1982, Job matching, coalition formation, and gross substitutes, Econometrica, 50, 1483-1504.

[14] Milgrom, P. and J. Roberts, 1990, Rationalizability, learning and equilibrium in games with strategic complementarities, Econometrica, 58, $1255-1278$.

[15] Milgrom, P. and C. Shannon, 1994, Monotone comparative statics, Econometrica, 62, 157-180.

[16] Pérez-Castrillo, D., 1994, Cooperative Outcomes through Noncooperative Games, Games and Economic Behavior 7, 428-440.

[17] Pérez-Castrillo, D. and D. Wettstein, 2001, Bidding for the Surplus: A Non-cooperative Approach to the Shapley Value, Journal of Economic Theory 100, 274-294.

[18] Pérez-Castrillo, D. and M. Sotomayor, 2002, A simple Selling and Buying Procedure, Journal of Economic Theory 103, 461-474.

[19] Salop, S., 1979, Monopolistic competition with outside goods, Bell. Journal of Economics, 10, 141-156.

[20] Shaked, A. and J. Sutton, 1982, Relaxing price competition though product differentiation, Review of Economic Studies, 49, 3-13.

[21] Shaked, A. and J. Sutton, 1983, Natural Oligopolies, Econometrica, 51, 1469-1484.

[22] Shapley, L.S., 1962, Complements and Substitutes in the Optimal Assignment Problem, Naval Research Logistics Quarterly, 9, 45-48. 
[23] Shapley, L.S., 1971, Cores of convex games, International Journal of Game Theory, 1, 11-26.

[24] Shapley, L.S. and M. Shubik, 1972, The assignment game I: The core, International Journal Game Theory, 1, 111-130.

[25] Tauman, Y., Urbano, A. and J. Watanabe, 1997, A Model of Multiproduct Price Competition, Journal of Economic Theory, 77, 377-401. 